\title{
Homeoprotein OTX1 and OTX2 involvement in rat myenteric neuron adaptation after DNBS-induced colitis
}

\author{
Michela Bistoletti ${ }^{\text {Equal first author, } 1}{ }^{\text {, Giovanni Micheloni }}{ }^{\text {Equal first author, } 1}{ }^{1}$, Nicolò Baranzini ${ }^{2}$, Annalisa Bosi ${ }^{1}$, Andrea Conti ${ }^{1}$, \\ Viviana Filpa ${ }^{1}$, Cristina Pirrone ${ }^{1}$, Giorgia Millefanti ${ }^{1}$, Elisabetta Moro ${ }^{3}$, Annalisa Grimaldi ${ }^{2}$, Roberto Valli ${ }^{1}$, \\ Andreina Baj ${ }^{1}$, Francesca Crema ${ }^{3}$, Cristina Giaroni ${ }^{\text {Corresp., }}{ }^{,}$, Giovanni Porta ${ }^{1}$ \\ 1 Department of Medicine and Surgery, University of Insubria, Varese, Italy \\ 2 Department of Biotechnology and Life Sciences, University of Insubria, Varese, Italy \\ 3 Department of Internal Medicine and Therapeutics, University of Pavia, Pavia, Italy \\ Corresponding Author: Cristina Giaroni \\ Email address: cristina.giaroni@uninsubria.it
}

Background Inflammatory bowel diseases are associated with remodeling of neuronal circuitries within the enteric nervous system, occurring also at sites distant from the acute site of inflammation and underlying disturbed intestinal functions. Homeoproteins orthodenticle OTX1 and OTX2 are neuronal transcription factors participating to adaptation during inflammation and underlying tumor growth both in the central nervous system and in the periphery. In this study, we evaluated OTX1 and OTX2 expression in the rat small intestine and distal colon myenteric plexus after intrarectal dinitro-benzene sulfonic (DNBS) acid-induced colitis.

Methods OTX1 and OTX2 distribution was immunohistochemically investigated in longitudinal muscle myenteric plexus (LMMP)-whole mount preparations. mRNAs and protein levels of both OTX1 and OTX2 were evaluated by qRT-PCR and Western blotting in LMMPs.

Results DNBS-treatment induced major gross morphology and histological alterations in the distal colon, while the number of myenteric neurons was significantly reduced both in the small intestine and colon. mRNA levels of the inflammatory markers, TNF $\alpha$, pro-IL1 $\beta$, IL6, HIF1 $\alpha$ and VEGF $\alpha$ and myeloperoxidase activity raised in both regions. In both small intestine and colon, an anti-OTX1 antibody labelled a small percentage of myenteric neurons, and prevalently enteric glial cells, as evidenced by co-staining with the glial marker S100ß. OTX2 immunoreactivity was present only in myenteric neurons and was highly colocalized with neuronal nitric oxide synthase. Both in the small intestine and distal colon, the number of OTX1- and OTX2-immunoreactive myenteric neurons significantly increased after DNBS treatment. In these conditions, OTX1 immunostaining was highly superimposable with inducible nitric oxide synthase in both regions. OTX1 and OTX2 mRNA and protein levels significantly enhanced in LMMP preparations of both regions after DNBS treatment.

Conclusions These data suggest that colitis up-regulates OTX1 and OTX2 in myenteric plexus both on site and distantly from the injury, potentially participating to inflammatory-related myenteric ganglia remodeling processes involving nitrergic transmission. 
1 Homeoprotein OTX1 and OTX2 involvement in rat 2 myenteric neuron adaptation after DNBS-induced colitis

3

4

5

Michela Bistoletti $1^{*}$, Giovanni Micheloni ${ }^{{ }^{*}}$, Nicolò Baranzini ${ }^{2}$, Annalisa Bosi ${ }^{1}$, Andrea Conti ${ }^{1}$, Viviana Filpa $^{1}$, Cristina Pirrone ${ }^{1}$, Giorgia Millefanti ${ }^{1}$, Elisabetta Moro $^{3}$, Annalisa Grimaldi ${ }^{2}$, Roberto Valli ${ }^{1}$, Andreina Baj ${ }^{1}$, Francesca Crema ${ }^{3}$,, Cristina Giaroni ${ }^{1}$, Giovanni Porta ${ }^{1 \#}$

${ }^{1}$ Department of Medicine and Surgery, University of Insubria, Varese, Italy

${ }^{2}$ Department of Biotechnology and Life Sciences, University of Insubria, Varese, Italy

${ }^{3}$ Department of Internal Medicine and Therapeutics, Section of Pharmacology, University of Pavia, Pavia, Italy

\section{Corresponding Author:}

Cristina Giaroni ${ }^{1}$

Via H. Dunant 5, Varese, 21100, Italy

Email address: cristina.giaroni@uninsubria.it

${ }^{*}$ These Authors equally contributed to this work

\#These Authors equally contributed to this work

\section{Abstract}

\section{Background}

Inflammatory bowel diseases are associated with remodeling of neuronal circuitries within the enteric nervous system, occurring also at sites distant from the acute site of inflammation and underlying disturbed intestinal functions. Homeoproteins orthodenticle OTX1 and OTX2 are neuronal transcription factors participating to adaptation during inflammation and underlying tumor growth both in the central nervous system and in the periphery. In this study, we evaluated OTX1 and OTX2 expression in the rat small intestine and distal colon myenteric plexus after intrarectal dinitro-benzene sulfonic (DNBS) acid-induced colitis.

\section{Methods} OTX1 and OTX2 distribution was immunohistochemically investigated in longitudinal muscle myenteric plexus (LMMP)-whole mount preparations. mRNAs and protein levels of both OTX1 and OTX2 were evaluated by qRT-PCR and Western blotting in LMMPs.

\section{Results}

DNBS-treatment induced major gross morphology and histological alterations in the distal colon, while the number of myenteric neurons was significantly reduced both in the small intestine and colon. mRNA levels of the inflammatory markers, TNF $\alpha$, pro-IL1 $\beta$, IL6, HIF1 $\alpha$ and VEGF $\alpha$ and myeloperoxidase activity raised in both regions. In both small intestine and colon, an antiOTX1 antibody labelled a small percentage of myenteric neurons, and prevalently enteric glial 
40 cells, as evidenced by co-staining with the glial marker S100 $\beta$. OTX2 immunoreactivity was

41 present only in myenteric neurons and was highly co-localized with neuronal nitric oxide

42 synthase. Both in the small intestine and distal colon, the number of OTX1- and OTX2-

43 immunoreactive myenteric neurons significantly increased after DNBS treatment. In these

44 conditions, OTX1 immunostaining was highly superimposable with inducible nitric oxide

45 synthase in both regions. OTX1 and OTX2 mRNA and protein levels significantly enhanced in

46 LMMP preparations of both regions after DNBS treatment.

\section{Conclusions}

48 These data suggest that colitis up-regulates OTX1 and OTX2 in myenteric plexus both on site

49 and distantly from the injury, potentially participating to inflammatory-related myenteric ganglia remodeling processes involving nitrergic transmission.

53

54

55

56

57

58

59

60

61

62

63

64

65

66

67

68

69

70

71

72

73

74

75

76

77

78

79

\section{Introduction}

Inflammatory bowel diseases (IBD) are chronic inflammatory disorders of the gastrointestinal tract with increasing incidence worldwide ( $\mathrm{Ng}$ et al., 2018). Inflammation develops because of an exaggerated immune response to luminal antigens derived from the gut microbiota or from infecting pathogens in genetically predisposed individuals (Ni et al., 2017; Baj et al., 2019a). Genetic and environmental factors also contribute to IBD development, although the exact aetiology remains to be fully disclosed. Inflammation leads to profound alterations of the intestinal architecture, which influence intrinsic neuronal circuitries constituting the enteric nervous system (ENS). Neuronal adaptation includes morphology, excitability and synaptic changes, with consequent alterations of sensory, motor and secretory functions contributing to the development of IBD-associated symptoms (Brierley, Linden 2014; De Schepper et al., 2008; Baj et al., 2019b). The cross-talk occurring among different cell populations of the enteric microenvironment (i.e. neurons, enteric glia, interstitial cells of Cajal, immunocytes), the saprophytic microbial flora and infiltrating inflammatory cells may account for the structural and functional changes occurring in enteric circuitries in different physiological and pathological conditions, including inflammation (Giaroni, 2015; Lomax, Fernández \& Sharkey, 2015; Bistoletti et al. 2019). For example, neuro-immune interactions may help explain the occurrence of damage at non-inflamed gastrointestinal sites, distant from the inflammatory process (Brierley, Linden 2014; De Schepper et al., 2008). It is, thus, particularly important to unveil possible molecular pathways sustaining neuronal degeneration during inflammatory states, both on site and, more distantly, along the gastrointestinal tract. Indeed, research in this field opens an exciting potential scenario where new molecules displaying high efficacy in the treatment of IBD may became available in association and/or substitution of conventional anti-inflammatory, immunosuppressive and biologic drugs (Pagano et al., 2016; Neurath, 2017; Szebeni et al., 2019; Pagano et al., 2019).

In the present study, we investigated possible changes in the expression of homeoprotein transcription factors, orthodenticle homeobox protein 1 and 2 (OTX1 and OTX2), in the rat myenteric plexus after an experimentally-induced colitis. OTX1 and OTX2 are transcription 
80 factors derived from homeobox-containing genes located, in humans, on chromosome 2p15 and 81 14q22, respectively (Kastury et al., 1994). OTX1 and OTX2 homeobox genes are the vertebrate 82 orthologues to the Drosophila orthodenticle homeobox genes and are essential for the 83 specification, regionalization and terminal differentiation of the rostral part of the central nervous 84 system (CNS) during development (Acampora et al., 2001; Larsen et al., 2010). OTX2 is an 85 essential factor influencing the maturation of various neuronal subpopulations, including 86 oculomotor, thalamic glutamatergic progenitors and midbrain dopaminergic neurons (Sherf et 87 al., 2015; Puelles et al., 2006). Recent evidence suggest for OTX2 a fundamental role in 88 regulating neuronal plasticity in the visual cortex (Bernard, Prochiantz, 2016). During development in both rodent and human, OTX1 is prevalently expressed in proliferative zones of the neocortex and is described to have a fundamental role in determining the volume and proportion of the visual cortex (Ando et al., 2008). Despite their central role in neuronal development and maturation, there are lines of evidence suggesting that both OTX1 and OTX2 are expressed also in adult tissues and may participate to the development of pathological conditions linked to inflammation (Housset et al., 2013; Azzolini et al., 2013). In addition, the involvement of both factors in tumor growth, such as in medulloblastoma, the most frequent malignant brain tumor in children, and in breast cancer has been demonstrated (Di et al., Terrinoni et al., 2011). Furthermore, OTX1 is involved in the epithelial damage promoting colorectal cancer progression (Yu et al., 2014). Recently, both OTX1 and OTX2 were found to be expressed in normal adult rat small intestine myenteric ganglia: OTX1 has been predominantly found in enteric glial cells and in few myenteric neurons, while OTX2 was uniquely expressed in the soma of a relatively small percentage of myenteric neurons (Filpa et al., 2017a). Expression of both orthodenticle homeobox proteins was upregulated during intestinal ischemia/reperfusion injury and correlated with alterations of the intestinal neuromuscular function in these pathophysiological conditions (Filpa et al., 2017a). Such changes involved an interplay between both transcription factors and enteric nitrergic neurons, sustaining the development of altered motor responses involving NO production (Filpa et al., 2017a). In our study, we aim for the first time to evaluate possible changes of OTX1 and OTX2 expression in rat colon myenteric ganglia, after dinitro-benzene sulfonic (DNBS) acid-induced colitis, by means of molecular biology and morphological approaches. In view of the possible adaptive changes induced by the inflammatory insult at remote sites from the injury, we also investigated the consequences of DNBS treatment on both OTX1 and OTX2 expression in small intestine myenteric plexus.

\section{Materials \& Methods}

\section{Animals}

Male Sprague-Dawley rats (weight 250-300 g, Envigo, Udine, Italy), were handled following principles of good laboratory animal care, in accordance with specific national and international laws and regulation. Animals were maintained at a regular 12/12-h light/dark cycle, under controlled environmental conditions (temperature $22 \pm 2^{\circ} \mathrm{C}$; relative humidity $60-70 \%$ ), with free 
120 access to a standard laboratory tap water and chow. . The protocol was approved by the Animal 121 Care and Use Ethics Committee of the University of Pavia (n. 3/2011).

122

123

124

125

126

127

128

129

130

131

132

133

134

135

136

137

138

139

140

141

142

143

144

145

146

147

148

149

150

151

152

153

154

155

156

157

\section{DNBS-induced colitis}

The model of DNBS-induced experimental colitis in rats was chosen since the inflammatory response develops rapidly, reaching the maximum grade in 6 days, and shares many features with the human IBDs response (Filpa et al., 2017b). A single dose (30 mg) of 2,4-dinitrobenzene-sulfonic acid (DNBS, ICN Biomedicals, CA, USA), dissolved in $0.25 \mathrm{ml}$ of $50 \%$ ethanol, was administered to isofluorane anaesthetized rats by means of a polyethylene (PE-60) catheter into the colon $8 \mathrm{~cm}$ proximal to the anus. Ethanol, able to break the mucosal barrier thus permitting DNBS penetration into the bowel wall, was administered $(0.25 \mathrm{ml})$ to control animals as a vehicle. . The dose of DNBS used in the study induces adequate inflammation, without causing unnecessary distress, suffering or mortality to the animals DNBS-treated and control rats were kept separated during the study. After 6 days, animals were euthanized and the small intestine and distal colon was removed and washed with a physiological Tyrode's solution (in $\mathrm{mM}: 137 \mathrm{NaCl} ; 2.68 \mathrm{KCl} ; 1.8 \mathrm{CaCl}_{2} .2 \mathrm{H}_{2} \mathrm{O} ; 2 \mathrm{MgCl}_{2} ; 0.47 \mathrm{NaH}_{2} \mathrm{PO}_{4} ; 11.9 \mathrm{NaHCO}_{3} ; 5.6$ glucose). . Throughout the treatment period, animals were monitored to evaluate possible physiological and behavioral changes (i.e., changes in body weight, respiration, occurrence of diarrhea, alterations of posture and in the appearance of the coat) as mark of suffering and distress.

\section{Assessment of colonic damage}

The severity of intestinal inflammation was evaluated macroscopically and histologically. Criteria for the macroscopic evaluation included the presence of adhesions between the colon and other intra-abdominal organs, the extension of hyperemia and macroscopic mucosal damage, the thickening of the colonic wall and the consistency of colonic faecal material, all attributed with a score ranging from 0 (no damage) to 6_(maximum damage) Filpa et al., (2017b. For the microscopic histological evaluation, standard hematoxylin and eosin (HE) staining was carried out on serial sections $(7 \mu \mathrm{m})$ of small intestine and colonic segments, from control and DNBS-treated rats, which were first fixed for $24-48 \mathrm{~h}$ with $4 \%$ formaldehyde in acetate buffer $0.05 \mathrm{M}$ and successively embedded in paraffin. Preparations were observed under a light microscope (Nikon Eclipse Ni; Nikon, Tokyo, Japan) and data were recorded using a DS-5M-L1 digital camera system (Nikon Corporation, Tokyo, Japan).

\section{Myeloperoxidase activity}

To evaluate the development of the inflammatory state caused by neutrophil infiltration, myeloperoxidase (MPO) was measured as previously described by Filpa et al. (2017a). Briefly, intestinal segments deprived of the mucosal layer were homogenized $(50 \mathrm{mg} / \mathrm{ml})$ with a solution 
158

159

160

161

162

163

164

165

166

167

168

169

170

171

172

173

174

175

176

177

178

179

180

181

182

183

184

185

186

187

188

189

190

191

192

193

194

195

196

of ice cold potassium phosphate buffer $(50 \mathrm{mM}, \mathrm{pH} 6.0)$ containing $0.5 \%$ hexadecyl

trimethylammonium bromide (HTAB. After centrifugation $\left(14000 \mathrm{rpm}, 20\right.$ minutes, $\left.4^{\circ} \mathrm{C}\right)$, an aliquot of the supernatant fraction was mixed with HTAB-phosphate buffer containing Odianisidine dihydrochloride with hydrogen peroxide before spectrophotometrically recording at $460 \mathrm{~nm}$ changes in the rate of absorbance. MPO activity was expressed in units (U)/wet tissue weight, where $U$ defined the amount of enzyme that degrades $1 \mu \mathrm{mol} / \mathrm{min}$ of hydrogen peroxide at $25^{\circ} \mathrm{C}$. Experiments were performed 6 times for each experimental group.

\section{Immunofluorescence}

Paraffin-embedded tissue sections and whole-mount preparations were processed for the immuno-localization of OTX1 and OTX2 on small intestine and colon of control and DNBStreated rats, as previously described in Bistoletti et al, 2019.

\section{Paraffin sections}

$7 \mu \mathrm{m}$ paraffin cross sections incubated with CD45, as a marker of inflammatory infiltration, or either with OTX1 or OTX2 with the pan neuronal marker HUC/D, for double staining. Optimal dilutions of antibodies are reported in Table 1. Coverslips were mounted with Citifluor mounting medium and then observed under a fluorescent microscope (Nikon Instruments).

\section{Whole-mount preparations}

Whole-mount preparation immunolabeling was performed on the longitudinal muscle with the attached myenteric plexus (LMMP) proceeding with a double-staining with optimally diluted primary and secondary antibodies (Table 1). Preparations were mounted onto glass slides with a mounting medium (Vectashield with DAPI; Vector Lab, Burlingame, CA, USA) and analyzed with Image J NIH image software (http://imagej.nih.gov/ij). Total neuron number per ganglion area was expressed as the ratio between the number of neurons positive for $\mathrm{HuC} / \mathrm{D}$ and the total ganglion area $\left(\mu \mathrm{m}^{2}\right)$. Areas of 15-19 myenteric ganglia from LMMP preparations of 5 vehicletreated CTR and 5 DNBS-treated animals were measured at 40x magnification by tracing boundaries around stained cell somas (HuC/D) (Filpa et al. 2017a). Neuronal cell body area was measured with Image J., The number of OTX1 or OTX2 immunoreactive neurons that colocalized with $\mathrm{HuC} / \mathrm{D}$ were counted from a total of 10-15 ganglia (5 animals for each experimental group) to establish the proportion of OTX1 and OTX2 expressing myenteric neurons, expressed as percentage of the total number of $\mathrm{HuC} / \mathrm{D}$ positive neurons (Filpa et al., 2017a). To evaluate negative controls and interference control staining, both primary and secondary antibody were omitted, incubating the colonic whole-mounts with non-immune serum from the same species in which the primary antibodies were raised. In all these conditions, no specific signal was detected. Pictures from preparations, collected with a Leica TCS SP5 confocal laser scanning system (Leica Microsystems GmbH, Wetzlar, Germany) were then processed with Adobe-Photoshop CS6S software.

\section{Real time quantitative RT-PCR}


197 To evaluate the influence of DNBS-induced colitis on OTX1, OTX2, TNF $\alpha$, pro-IL1 $\beta$, IL6,

198

199

200

201

202

203

204

205

206

207

208

209

210

211

212

213

214

215

216

217

218

219

220

221

222

223

224

225

226

227

228

229

230

231

232

233

HIF $1 \alpha$, VEGF $\alpha$, mRNA levels, total RNA from rat small intestine and colon LMMPs was extracted with TRIzol (Invitrogen) and treated with DNase I (DNase Free, Ambion) to remove possible traces of contaminating DNA. $2.5 \mu \mathrm{g}$ of total RNA was retrotranscribed using the High Capacity cDNA synthesis kit (Applied Biosystems, Life Technologies, Grand Island, NY, USA).Quantitative RT-PCR was performed on the Abi Prism 7000 real-time thermocycler (Applied Biosystems) with Power Sybr Green Universal PCR Master Mix (Applied Biosystems)

following manufacturer's instructions. Primers were designed to have a similar amplicon size and similar amplification efficiency as required for the utilization of the $2^{-\Delta \Delta C t}$ method to compare gene expression (Bin et al., 2018), using Primer Express software (Applied Biosystems) on the basis of available sequences deposited in public database (Table 2). For quantitative RT-PCR a final concentration of $500 \mathrm{nM}$ for each primer was used. Experiments were performed at least 7 times for each experimental group. $2^{-\Delta \Delta C t}$ values obtained from the comparison between normalized Ct values of DNBS- treated samples with those obtained from control samples were used to evaluate the effect of DNBS-induced colitis on OTX1 and OTX2 mRNA levels in the small intestine and colon.

\section{Western immunoblot analysis}

Purified membrane fractions obtained after successive centrifugations of homogenized intestinal LMMPs preparations were used to analyze OTX1 and OTX2 protein level as described elsewhere (Giaroni et al., 2011). Membrane incubation with OTX1 and OTX2 primary antibodies was performed by incubation with a horseradish peroxidase-conjugated secondary antisera (Table 1). The signal of antibody/substrate complex was visualized by chemiluminescence using an enhanced chemiluminescence kit (ECL advance Amersham Pharmacia Biotech, Cologno Monzese, Italy), and then evaluated by densitometric analysis using Image J NIH image software. The effect of DNBS-induced colitis on OTX1 and OTX2 protein levels was expressed as the percentage variation of the optical density (expressed in arbitrary units) of OTX1 and OTX2 signals normalized to the respective $\beta$-actin, used as protein loading control, in LMMP preparations obtained from DNBS-treated animals compared to controls. Experiments were performed at least 5 times for each experimental group. Specificity of OTX1 and OTX2 primary antibodies was evaluated by testing their selectivity in spontaneous immortalized human Müller cell line (MIO-M1) and in rat hippocampus, respectively (data not shown) (Filpa et al., 2017). Negative controls were performed by omitting the primary antibody.

\section{Statistical analysis}

All data are expressed as mean \pm S.E.M. Statistical significance was calculated by either Student's $\mathrm{t}$ test or by one-way ANOVA with Tukey's post hoc test, where appropriate, using GraphPad 
234 Prism (version 5.3 GraphPad software, San Diego, CA, USA). Differences among groups were 235 considered significant when $\mathrm{P}$ value were 0.05 or lower.

236

237 Results

238 Assessment of colitis

239 Macroscopic assessment

240 Body weight was significantly reduced in DNBS-treated rats compared to non-inflamed controls 241 at day 6 after treatment (DNBS-treated: $280 \pm 6.50 \mathrm{~g}, \mathrm{n}=7$; vehicle-treated CTR: $306 \pm 2.3 \mathrm{~g}, \mathrm{n}=7$,

$242 \mathrm{P}=0.027$ by Student's $t$ test). Six days after DNBS administration, the distal colon was thickened 243 and ulcerated with evident regions of transmural inflammation, adhesions between the colon and 244 other intra-abdominal organs were often present and the bowel was occasionally dilated.

245 Macroscopic damage score significantly increased in the colon of DNBS-treated animals in 246 comparison with vehicle-treated controls (DNBS-treated: $4.80 \pm 021, \mathrm{n}=10$; CTR: $0.3 \pm 0.08, \mathrm{n}=14$ $247 \mathrm{P}<0.0001$, by Student's $t$ test). No significant gross morphology changes were observed in the 248 small intestine. This was reflected by low macroscopic damage scores in both experimental 249 groups (DNBS-treated: $0.11 \pm 0.08, \mathrm{n}=10$; CTR: $0.09 \pm 0.05, \mathrm{n}=14$, by Student's $\mathrm{t}$ test). These 250 findings are in agreement with other reports, showing that after induction of an experimentally251 induced colitis, the occurrence of gross macroscopic changes is obvious only on the site of 252 253

254

255

256

257

258

259

260

261

262

263

264

265

266

267

268

269

270

271

272 injury, preserving distant sites along the gastrointestinal tract (Mourad et al., 2016).

\section{Histological assessment}

A further set of experiments was carried out on intestinal cross sections to evaluate the impact of DNBS-induced colitis on the architecture of the rat small intestine and distal colon by means of standard HE staining, and the degree of inflammatory infiltrate by CD45 immunofluorescent staining. Small intestine and distal colon cross-sections obtained from control animals showed normal histological features, with compact myenteric ganglia, formed by healthy neuron and glial cells, laying between the circular and longitudinal muscle of the muscularis propria (Fig. 1, panels A, B and K, L). CD45 staining, indicative of inflammatory cell infiltration, was almost negligible in both small intestine and colon of control cross sections (Fig. 1 panels, $\mathbf{C}$ and $\mathbf{M}$ ). At day six after DNBS treatment, small intestine specimens did not show prominent histological abnormalities (Fig 1, panels F, G). In contrast, both mucosa and serosal epithelium of the distal colon obtained from DNBS-treated rats, displayed morphological abnormalities (Fig. 1, panels P-R). The colonic mucosal surface was irregular and crypt architecture was profoundly altered, the muscularis mucosae, submucosa and muscularis propria layers were thickened (Fig. 1, panel P). Prominent spaces between smooth muscle cells and leukocyte infiltration were also observed (Fig. 1, panel Q). In the small intestine of DNBS-treated animals myenteric neurons had a normal morphology comparable to that observed in control preparations as shown in panel $\mathbf{G}$ of Figure 1. In contrast, colonic myenteric ganglia underwent important degenerative changes, with neurons displaying cytoplasm vacuolization and irregular nuclear and cellular membrane. Large 
273 spaces between muscle cells were also evident (Fig. 1, panel R). After DNBS-treatment, CD45

274 staining significantly increased both in the small intestine and in the colon (Fig. 1 panels, $\mathbf{H}$ and 275 S).

276 We then focused our histological investigations on myenteric ganglia by carrying out 277 immunofluorescence staining of small intestine and distal colon LMMP whole-mount

278 preparations with the pan neuronal marker $\mathrm{HuC} / \mathrm{D}$. In this set of experiments changes in 279 myenteric neuron number were observed in both gastrointestinal regions after DNBS treatment 280 (Fig. 2 panels A-F). In particular, the number of myenteric neurons staining for $\mathrm{HuC} / \mathrm{D}$ was 281 significantly reduced ( $\mathrm{P}<0.05$, by one way-ANOVA with Tukey's post hoc test) with respect to 282 values obtained in respective control preparations in both regions (Fig 2 panel $\mathbf{E}$ ). Myenteric 283 neuron number was reduced to a significantly greater extent $(\mathrm{P}<0.05$, by one way-ANOVA with 284 Tukey's post hoc test) in the colon than in the small intestine (Fig 2 panel E). In addition, in the 285 286

287

288

289

290

291

292

293

294

295 296 colon, but not in the small intestine, DNBS-induced inflammation was associated with a significant reduction of myenteric neuron soma area $(\mathrm{P}<0.001$, by one way-ANOVA with Tukey's post hoc test) compared to control preparations (Fig 2, panel F). As expected, in accordance with previous studies, in control preparations, in the distal colon the number of myenteric neurons was significantly lower $(\mathrm{P}<0.05$, by one way-ANOVA with Tukey's post hoc test) than in the small intestine (Fig 2, panel E). Additionally, in the distal colon the neuronal soma area was significantly larger $(\mathrm{P}<0.05$, by one way-ANOVA with Tukey's post hoc test) than in the small intestine (Fig 2, panel F). Overall, these observations suggest that after DNBSinduced colitis, major histological changes involving myenteric ganglia, occur in the distal colon. However, also the small intestine may be influenced by the injury, as indicated by the increased infiltration of inflammatory $\mathrm{CD} 45^{+}$cells and by the reduction of myenteric neuron number.

\section{Biomolecular assessment}

The occurrence of an inflammatory response in LMMP preparations, on site and distantly from the injury, was further investigated by evaluating the levels of the mRNA of different cytokines, which are considered standard markers of intestinal inflammation, and by measuring MPO activity. In both intestinal regions, after DNBS treatment, a significant enhancement of the expression of inflammatory cytokines TNF $\alpha(\mathrm{P}<0.001$ in both regions by one-way ANOVA with Tukey's post hoc test), pro-IL1 $\beta(\mathrm{P}<0.01$ in the ileum and $\mathrm{P}<0.001$ in the colon by one wayANOVA with Tukey's post hoc test), IL6 ( $\mathrm{P}<0.001$ in both regions by one-way ANOVA with Tukey's post hoc test), and of HIF- $1 \alpha(\mathrm{P}<0.05$ in both regions by one-way ANOVA with Tukey's post hoc test) and VEGF $\alpha(\mathrm{P}<0.01$ in both regions by one-way ANOVA with Tukey's post hoc test), was evidenced with respect to control animals (Fig 3, panels A-E). As regards the pro-inflammatory cytokines, enhancement of TNF $\alpha$ mRNA was similar in the colon and in the small intestine, while pro-IL $1 \beta$ and IL-6 mRNA levels were significantly higher in the colon with respect to the small intestine ( $\mathrm{P}<0.05$ and $\mathrm{P}<0.001$, respectively by one-way ANOVA with Tukey's post hoc test) (Fig 1, panels A-C). HIF $1 \alpha$ and VEGF $\alpha$ mRNA levels after DNBS 
312 treatment was not significantly different in the small intestine with respect to the distal colon

313 (Fig 1, panels D-E).

314 Myeloperoxidase (MPO) activity significantly increased in rat small intestine $(\mathrm{P}<0.001$, by one

315 way-ANOVA with Tukey's post hoc test) and colonic $(\mathrm{P}<0.001$, by one way-ANOVA with

316 Tukey's post hoc test) segments compared to control animals, suggesting the occurrence of

317 inflammation-induced neutrophil infiltration on site and distally from the injury (Fig 1, panel F).

318

Distribution of OTX1 immunoreactivity in small intestine and colon LMMP whole-mount

320 preparations from normal and DNBS-treated animals

321

322

In view of the adaptive changes observed after DNBS-induced inflammation in the rat myenteric plexus, we subsequently evaluated if this adaptation encompasses alterations in the distribution of homeobox proteins involved neuronal plasticity, OTX1 and OTX2, in cross sections and

324

325

326

327

328

329

330

331

332

333

334 LMMP whole-mounts of the small intestine and distal colon. In cross-sections of small intestine and distal colon obtained from vehicle-treated control rats, myenteric ganglia displayed faint OTX1 immunoreactivity (Fig 1, panels $\mathbf{D}$ and $\mathbf{N}$ ). In control LMMP whole-mount preparations OTX1 antibody stained few myenteric neurons in both the small intestine $(3.79 \pm 1.00 \%, \mathrm{n}=15)$ and distal colon $(4.77 \pm 1.43 \%, \mathrm{n}=15)$ (Fig 4, panel A). In both intestinal regions, OTX1 antibody stained the soma and nucleus of large and medium size myenteric neurons with either a round or an ovoidal shape (Fig 5 panels A-C, and G-I). In small intestine and distal colon cross-sections obtained from DNBS-treated animals, a significant increase of OTX1 staining was observed within the myenteric plexus with respect to control preparations (Fig 1, panels I and T). After DNBS treatment, a significant increase in the number of OTX1 immunoreactive myenteric neurons was observed in whole-mount preparations of both small intestine $(9.86 \pm 0.86 \%, \mathrm{n}=15$, $\mathrm{P}<0.0001$ by one-way ANOVA with Tukey's post hoc test) and colon $(40.25 \pm 1.2 \%, \mathrm{n}=15$ $\mathrm{P}<0.0001$, by one-way ANOVA with Tukey's post hoc test) with respect to control preparations (Fig 4, panel A) (Fig 5 panels D-F and J-L). In DNBS-treated colonic whole mount preparations, the percentage of OTX1 immunoreactive myenteric neurons was significantly higher than in the small intestine (Fig 4, panel A). In LMMP whole-mount preparations of both

340 intestinal regions, OTX1 antibody co-localized with the glial marker S100 $\beta$, and co-staining was particularly intense after DNBS-induced inflammation (Fig. 5 panels M-R). In small intestine and colonic LMMPs obtained from control and DNBS-treated animals, myenteric neurons staining for OTX1 were also immunopositive for iNOS, as shown in panels A-L of Figure 6. Both in control and DNBS-treated LMMP whole-mount preparations, OTX1 immunostaining did not co-localize with nNOS (Fig 6 panels M-O). These results suggest that OTX1 is expressed both in myenteric neurons, where it is highly co-expressed with iNOS, and in enteric glial cells of the rat small intestine and distal colon. After DNBS-induced colitis the number of myenteric neurons expressing OTX1 is highly upregulated both on site and distantly.

Distribution of OTX2 immunoreactivity in small intestine and colon LMMP whole-mount preparations from normal and DNBS-treated animals

352 In control sections of the rat small intestine and colon, faint OTX2 staining was found in 
355

356

357

358

359

360

361

362

363

364

365

366

367

368

369

370

371

372

373

374

375

376

377

378

379

380

381

382

383

384

385

386

387

388

389

390

391

392

393

394

small intestine and in the distal colon (Fig 7 panels A-C and G-I, respectively). Some neurons had a large cell body, while other smaller neurons with an ovoidal shape were, prevalently, localized in the periphery of the ganglion. The percentage of OTX2-immunoreactive neurons in control small intestine and distal colon myenteric plexus was $7.07 \pm 1.68 \%, \mathrm{n}=15$ and $8.93 \pm 2.11 \%, \mathrm{n}=15$, respectively (Fig 4, panel B). In small intestine and colon cross-sections obtained from DNBS-treated animals, OTX2 staining significantly increased in myenteric ganglia with respect to control preparations (Fig 1, panels $\mathbf{J}$ and $\mathbf{U}$ ). Accordingly, after DNBS treatment, the percentage of OTX2 immunoreactive myenteric neurons in LMMP whole-mount preparations obtained from the small intestine and colon significantly increased $(47.95 \pm 3.20 \%$, $\mathrm{n}=15 ; 41.47 \pm 5.12 \%, \mathrm{n}=15$, respectively $\mathrm{P}<0.001$, by one-way ANOVA with Tukey's post hoc test) with respect to control values, (Fig 4, panel B and Fig 7 panels D-F and J-L). The number of OTX2 immunoreactive neurons was not significantly different in the two regions (Fig 4, panel B). In small intestine and colonic myenteric ganglion obtained from control and DNBS-treated animals, the majority of OTX2 immunoreactive neurons stained for nNOS (Fig 8, panels A-L). There was no evidence of co-staining between OTX2 and iNOS, as shown in panels M-O of Figure 8. In small intestine and distal colon LMMPs obtained from control animals $31.94 \pm 3.33 \%, \mathrm{n}=10$ and $30.83 \pm 10.83 \%, \mathrm{n}=10$, respectively, of OTX2-IR myenteric neurons stained also for OTX1. In both regions, after DNBS treatment, $25.82 \pm 5.78 \%, \mathrm{n}=10$ and $25.63 \pm 2.17 \% \mathrm{n}=10$, respectively, of OTX2-IR were also immunoreactive for OTX1. These findings suggest that OTX2 is exclusively expressed in myenteric neurons and displays a high co-localization with nNOS. Experimentally-induced colitis upregulates the number of myenteric neurons expressing OTX2 both on site and distantly.

\section{DNBS-induced colitis upregulates OTX1 mRNA and protein in LMMPs of the rat small intestine and distal colon}

In order to confirm the immunofluorescence data reflecting an up-regulation of OTX1 and OTX2 in the rat small intestine and colon myenteric plexus after DNBS-induced colitis, we evaluated the levels of mRNA and proteins of both homeobox proteins in LMMP preparations. Rat small intestine and colonic OTX1 mRNA levels obtained in the different experimental groups are described in panel A of Figure 9. In LMMPs of both regions, DNBS treatment induced a significant increase of OTX1 mRNA with respect to values obtained in control preparations (small intestine: $\mathrm{P}<0.05$; distal colon: $\mathrm{P}<0.001$, by one-way ANOVA with Tukey's post hoc test). OTX1 mRNA enhancement in colonic specimens was significantly higher than in the small intestine ( $\mathrm{P}<0.001$, by one-way ANOVA with Tukey's post hoc test). In rat small intestine and colonic LMMP preparations the specific OTX1 antibody revealed one band at $37 \mathrm{kDa}$ (Fig 9, panel B). In both gut regions, after DNBS treatment, OTX1 protein expression significantly increased (small intestine: $\mathrm{P}<0.05$; distal colon: $\mathrm{P}<0.01$, by one-way ANOVA with Tukey's post hoc test). These biomolecular data confirm that an experimentally-induced colitis may upregulate both the transcript and protein of OTX1 in the myenteric plexus both on site and distantly from the injury. 
396

397

398

399

400

401

402

403

404

405

406

407

408

409

410

411

412

413

414

415

416

417

418

419

420

421

422

423

424

425

426

427

428

429

430

431

432

433

434

\section{DNBS-induced colitis upregulates OTX2 $\mathrm{mRNA}$ and protein in LMMPs of the rat small} intestine and distal colon

OTX2 mRNA levels measured in rat small intestine and colonic LMMPs preparations obtained from control and DNBS-treated animals are described in panel $\mathbf{C}$ of Figure 9. In both regions, DNBS treatment induced a significant increase of OTX2 mRNA with respect to values obtained in control preparations (small intestine: $\mathrm{P}<0.05$; distal colon: $\mathrm{P}<0.001$, by one-way ANOVA with Tukey's post hoc test). OTX2 mRNA levels in small intestine specimens were not significantly different than those obtained in the distal colon. In rat small intestine and colon LMMP preparations the specific OTX2 antibody revealed one band at $32 \mathrm{kDa}$ (Fig 9, panel D). In both gut regions, OTX2 protein expression significantly increased ( $\mathrm{P}<0.01$, by one-way ANOVA with Tukey's post hoc test) after DNBS treatment. After DNBS-induced colitis, analogously to OTX1, up-regulation of OTX2 in the rat myenteric plexus was evidenced also for the transcript and protein, by means of qRT-PCR and western immunoblotting.

\section{Discussion}

In myenteric neurons, intestinal inflammation induces adaptive changes, which may be responsible for both acute and long-lasting alterations of the gastrointestinal functions, underlying disease symptoms (Brierley, Linden 2014). The molecular mechanism/s of enteric neuronal adaptation to inflammation are still largely to be uncovered. In this study, we show that the expression of orthodenticle homeoproteins, OTX1 and OTX2, which participate to neuroplastic changes both in physiological and pathological conditions (Spatazza et al., 2013), is up-regulated in the myenteric plexus of the rat small intestine and distal colon after an experimentally induced colitis with dinitro-benzene sulfonic (DNBS) acid. In analogy with the results recently obtained in the rat small intestine myenteric plexus (Filpa et al., 2017a), in the present study OTX1-immunoreactivity (IR) is predominantly found in enteric glial cells and in few neurons of the rat distal colon myenteric plexus, while OTX2-IR is uniquely detected in the soma of a relatively small percentage of myenteric neurons. In pathophysiological conditions, i.e. after in vivo-induced ischemia/reperfusion (I/R) injury as well as in mild inflammatory conditions induced in sham-operated animals OTX1 and OTX2 expression in rat small intestine myenteric ganglia significantly enhanced (Filpa et al., 2017a).. In good agreement, in the present study we show a significant increase of both OTX1 and OTX2 mRNA and protein levels in longitudinal muscle myenteric plexus (LMMP) preparations of the rat small intestine and distal colon myenteric plexus after DNBS-induced colitis. In addition, the number of myenteric neurons staining for both transcription factors in the two intestinal regions significantly increases, suggesting that the inflammation influences the expression of both homeoproteins in myenteric neurons, on site and distantly from the injury. This adaptive response is not associated with major histopathological alterations in the small intestine myenteric plexus. In line with previous data,DNBS treatment, induces important morphological and histological changes, including distortion of myenteric ganglia with signs of neuronal 
435 degeneration, in the colon, but not in the small intestine, where, however, the number of 436 myenteric neurons significantly decreases. Interestingly, MPO activity and the number of CD45 437 positive cells, which are indicative of inflammatory infiltration, are elevated both in small 438 intestine and distal colon segments. In addition, after DNBS-induced colitis, in rat colonic and 439 small intestine LMMPs the levels of pro-inflammatory cytokines, such as TNF $\alpha$, pro-IL1 $\beta$ and 440 IL6, and of factors involved in active intestinal inflammation, such as HIF $1 \alpha$ and VEGF $\alpha$, are

441

442

443

444

445

446

447

448

449

450

451

452

453

454

455

456

457

458

459

460

461

462

463

464

465

466

467

468

469

470

471

472

473

474 up-regulated, as observed along the rat gastrointestinal tract after trinitrobenzene sulfonic(TNBS) acid- and DNBS-induced colitis by other groups ( Bakirtzi et al., 2014; Barada et al., 2006). Overall, our observations suggest that important molecular changes, including upregulation of OTX1 and OTX2 pathways, may occur in the myenteric plexus along the gastrointestinal tract in response to an inflammatory challenge. Such changes may be transmitted from the site of injury to distant sites, where overt structural changes are not evident, and may account for the functional alterations observed in the proximal intestine in both IBD patients and animal models, including altered motility (Brierley, Linden, 2014; De Schepper et al., 2008; Blandizzi et al., 2003). We cannot exclude that inflammation-induced OTX1 and OTX2 up-regulation in rat small intestine and distal colon myenteric neurons may influence the neuromuscular function by regulating enteric neurotransmitter pathways. Indeed, TNBS-induced colitis in the rat was associated with a decrease of neurotransmitter release, not only in the inflamed colon, but also distally, in the small intestine resulting in the reduction of small intestinal transit (Blandizzi et al., 2003). Nitric oxide (NO), may represent a putative enteric neurotransmitter involved in OTX1 and OTX2 inflammation-induced upregulation. Noteworthy, a preferential involvement of enteric nitrergic pathways underlays development of dysmotility after deletion of a homeobox gene phylogenetically related to OTX, such as Ncx/Hox11L.1 (Kobayashi et al., 2007). In addition, the occurrence of an interplay between enteric nitrergic pathways and OTX transcription factors has been suggested to sustain nitric oxide (NO)-mediated dysmotility after I/R injury in the gut (Filpa et al., 2017a). In these conditions, , NO was shown to favor OTX1 and OTX2 up-regulation in rat small intestine myenteric ganglia. After I/R, NO derived from iNOS promoted OTX1 up-regulation more, while nNOS more closely related to OTX2 upregulation. During gut inflammation and I/R injury, iNOS and nNOS have different roles on enteric neuronal homeostasis, retaining a neurodamaging and neuroprotective role, respectively (Rivera et al., 2012; Filpa et al., 2017; Bódi et al., 2019). Activation of the inducible isoform and downregulation of nNOS, are correlated with derangement of the neuromuscular function and slowing of the gastrointestinal transit (Rivera et al., 2012; Giaroni et al., 2013; Filpa et al., 2015). In view of the relationship between the two NO synthases and OTX1 and OTX2 these effects on intestinal motility may involve corresponding activation of molecular pathways downstream of OTX1 and OTX2, respectively (Filpa et al., 2017). Accordingly, in the present study, a substantial involvement of iNOS and nNOS in colitis-induced upregulation of OTX1 and OTX2, respectively, is suggested by the superimposition of OTX1 with iNOS and OTX2 with nNOS immunostaining, in myenteric ganglia of both regions studied. It is possible that activation of the iNOS-OTX1 pathway both in neurons and glial cells may sustain inflammation-induced 
475 alterations of the enteric neuromuscular function, as observed after I/R injury (Filpa et al., 476 2017a). Interestingly, overexpression of OTX1 sustained human colorectal cancer cell 477 proliferation and invasion in vitro and tumor growth in vivo, suggesting a pathogenic role for this 478 homeoprotein in colon cancer (Yu et al., 2014). By contrast, up-regulation of OTX2 in nNOS 479 myenteric neurons may have a protective role, preventing inflammation-induced neuronal cell 480 death, as suggested for OTX2 expression in photoreceptors (Housset et al., 2013). Different 481 hypotheses may be put forward to explain the mechanism/s underlying NO-mediated up482 regulation of OTX1 and OTX2. This latter may depend on increased levels of NO-derived 483 reactive oxygen species (ROS), whose production in myenteric neurons enhances as a 484 consequence of several gut pathophysiological conditions ( Carpanese et al., 2014; Thrasivoulou 485 et al., 2006). In this context, OTX2 up-regulation was recently observed in bovine in vitro 486 embryos as a result of increased oxidative stress inducing ROS production (Leite et al., 2017). 487 We cannot, however, exclude that OTX1 and OTX2 up-regulation in our model may depend 488 upon neuroimmune interactions between different subpopulation of enteric neurons and 489 immunocytes (De Schepper et al., 2008). Neuronal cells in the ENS are located in close

490

491

492

493

494

495

496

497

498

499

500

501

502

503

504

505

506

507

508

509

510

511

512

513 proximity to mucosal immunocytes and may regulate one another's functions by releasing a complex set of cytokines, neurotransmitters and hormones (Lakhan, Kirchgessner, 2010). OTX1 and OTX2 expression in the colonic myenteric plexus may be influenced by inflammatory mediators, such as VEGF $\alpha$, which is positively correlated to OTX2 expression in retinal pigment epithelial cells during inflammation (Azzolini et al., 2013). More recently, TNF $\alpha$ has been proposed as a modulator of OTX2 expression in in vitro models of chronic subretinal inflammation (Mathis et al., 2017). In addition, a recent genomewide study has identified a positive correlation between inflammatory cytokines, particularly IL6, and OTX1, in the pathogenesis of foot-and-mouth viral disease in animals (Zhang et al., 2018). Circulating cytokines and inflammatory mediators may influence myenteric neurons even at sites distant from the lesion, thus sustaining OTX1 and OTX2 up-regulation in the small intestine myenteric plexus (Liu et al., 2000). Changes in myenteric neuron responses during inflammation may be conveyed also via neuronal pathways constituting local or extrinsic reflex arches, as demonstrated in gastroparesis associated with TNBS-induced ileitis in rats (Moreels et al., 2001). OTX proteins may be secreted and internalized by live cells, including neurons, and a retrograde transfer of these proteins from colonic myenteric neurons to those of the small intestine by peculiar nonconventional mechanisms is also feasible (Joshi et al., 2011).

The pathophysiology of intestinal inflammation reflects a balance between mucosal injury and repair mechanism and may have important consequences on the integrity of intrinsic neuronal circuitries constituting the ENS. Several studies are focused on molecular pathways underpinning damage in different cellular populations, including myenteric neurons. Development of new therapeutic strategies in this field comprise the modulation of inflammation-induced cell death pathways (Baj et al., 2019a and b). In this view our data, by providing new hints on possible regulatory genes involved in myenteric neurons response to an 
514 inflammatory injury may contribute to the development of novel strategies for the treatment of

515 gastrointestinal diseases with important social and clinical impact, such as IBD.

516

517 Conclusions

518 In this study we provide evidence that DNBS treatment in rats may elevate inflammatory

519 markers not only in the site of inflammation, but also distally, in the small intestine. Such

520 response is associated with changes in myenteric neuron number in both regions, although more

521 severe damage occurs in the distal colon. After DNBS treatment the expression of two

522 homeobox transcription factors, OTX1 and OTX2, is upregulated in the myenteric plexus of the

523 small intestine and distal colon.

524

525

Acknowledgements

526 The Authors wish to thank Mr Antonio Pelizzoli for the technical support. Dr Fabrizio

527 Bolognese, Dr Ivan Vaghi, are kindly acknowledged for the excellent assistance in the

528 acquisition of confocal images. Michela Bistoletti, Giovanni Micheloni and Annalisa Bosi are

529 PhD students of the "Experimental and Translational Medicine" course at the University of

530 Insubria. Nicolò Baranzini is a PhD student of the Biotechnology, Biosciences and Surgical

531 Technology" course at the University of Insubria.

532

533 References

534 Acampora D, Boyl PP, Martinez-Barbera JP, Annino A, Signore M, Simeone A. 2001. Otx

535 genes in evolution: are they involved in instructing the vertebrate brain morphology? Journal of 536 Anatomy 199:53-62.

537 Ando K, Yagi H, Suda Y, Aizawa S, Sakashita M, Nagano T, Terashima T, Sato M. 2008.

538 Establishment of framework of the cortical area is influenced by Otx1. Neuroscience Research 539 60(4):457-459.

540 Azzolini C, Pagani IS, Pirrone C, Borroni D, Donati S, Al Oum M, Pigni D, Chiaravalli AM, 541 Vinciguerra R, Simonelli F, Porta G. 2013. Expression of VEGF-A, Otx homeobox and p53

542 family genes in proliferative vitreoretinopathy. Mediators of Inflammation 857380.

543 Baj A, Moro E, Bistoletti M, Orlandi V, Crema F, Giaroni C. 2019a. Glutamatergic Signaling

544 Along The Microbiota-Gut-Brain Axis. International Journal of Molecular Science 20(6). pii: 545 E1482.

546 Baj A, Bistoletti M, Bosi A, Moro E, Giaroni C, Crema F. 2019b. Marine Toxins and 547 Nociception: Potential Therapeutic Use in the Treatment of Visceral Pain Associated with 548 Gastrointestinal Disorders. Toxins 11:8.

549 Bakirtzi K, West G, Fiocchi C, Law IK, Iliopoulos D, Pothoulakis C. 2014. The neurotensin550 HIF-1 $\alpha$-VEGF $\alpha$ axis orchestrates hypoxia, colonic inflammation, and intestinal angiogenesis. 551 American Journal of Pathology 184(12):3405-3414. 
552

553

554

555

556

557

558

559

560

561

562

563

564

565

566

567

568

569

570

571

572

573

574

575

576

577

578

579

580

581

582

583

584

585

586

587

588

589

590

591

Barada KA, Mourad FH, Sawah SI, Khoury C, Safieh-Garabedian B, Nassar CF, Saadé NE. 2006. Localized colonic inflammation increases cytokine levels in distant small intestinal segments in the rat. Life Science Oct 79(21):2032-2042.

Bernard C, Prochiantz A. 2016. Otx2-PNN Interaction to Regulate Cortical Plasticity. Neural Plasticity 7931693.

Bin A, Caputi V, Bistoletti M, Montopoli M, Colucci R, Antonioli L, De Martin S, Castagliuolo I, Orso G, Giaroni C, Debetto P, Giron MC. 2018. The ecto-enzymes CD73 and adenosine deaminase modulate 5'-AMP-derived adenosine in myofibroblasts of the rat small intestine. Purinergic Signalling 14(4):409-421.

Bistoletti M, Caputi V, Baranzini N, Marchesi N, Filpa V, Marsilio I, Cerantola S, Terova G, Baj A, Grimaldi A, Pascale A, Frigo G, Crema F, Giron MC, Giaroni C. 2019. Antibiotic treatment-induced dysbiosis differently affects BDNF and TrkB expression in the brain and in the gut of juvenile mice. PLoS One 14(2):e0212856.

Blandizzi C, Fornai M, Colucci R, Baschiera F, Barbara G, De Giorgio R, De Ponti F, Breschi MC, Del Tacca M. 2003. Altered prejunctional modulation of intestinal cholinergic and noradrenergic pathways by alpha2-adrenoceptors in the presence of experimental colitis. British Journal of Pharmacology 139(2):309-20.

Bódi N, Szalai Z, Bagyánszki M. 2019. Nitrergic Enteric Neurons in Health and Disease-Focus on Animal Models. International Journal of Molecular Science 20(8). pii: E2003.

Brierley SM, Linden DR. 2014. Neuroplasticity and dysfunction after gastrointestinal inflammation. Nature Reviews in Gastroenterology and Hepatology 11(10):611-627.

Carpanese E, Moretto P, Filpa V, Marchet S, Moro E, Crema F, Frigo G, Giaroni C. 2014 Antagonism of ionotropic glutamate receptors attenuates chemical ischemia-induced injury in rat primary cultured myenteric ganglia. PLoS One 9(11):e113613.

Ceccotti C, Giaroni C, Bistoletti M, Viola M, Crema F, Terova G. 2018. Neurochemical characterization of myenteric neurons in the juvenile gilthead sea bream (Sparus aurata) intestine. PLoS One 13(8):e0201760.

De Schepper HU, De Man JG, Moreels TG, Pelckmans PA, De Winter BY. 2008 Gastrointestinal sensory and motor disturbances in inflammatory bowel disease - clinical relevance and pathophysiological mechanisms. Alimentary Pharmacology and Therapeutics 27(8):621-637.

Di C, Liao S, Adamson DC, Parrett TJ, Broderick DK, Shi Q, Lengauer C, Cummins JM, Velculescu VE, Fults DW, McLendon RE, Bigner DD, Yan H. 2005. Identification of OTX2 as a medulloblastoma oncogene whose product can be targeted by all-trans retinoic acid. Cancer Research 65(3):919-924.

Filpa V, Carpanese E, Marchet S, Prandoni V, Moro E, Lecchini S, Frigo G, Giaroni C, Crema F. 2015. Interaction between NMDA glutamatergic and nitrergic enteric pathways during in vitro ischemia and reperfusion. European Journal of Pharmacology 750:123-131.Filpa V, Carpanese E, Marchet S, Pirrone C, Conti A, Rainero A, Moro E, Chiaravalli AM, Zucchi I, Moriondo A, Negrini D, Crema F, Frigo G, Giaroni C, Porta G. 2017a. Nitric oxide regulates homeoprotein OTX1 and OTX2 expression in the rat myenteric plexus after intestinal ischemia- 
592 reperfusion injury. American Journal of Physiology Gastrointestinal and Liver Physiology 593 312(4):G374-G389.

594 Filpa V, Bistoletti M, Caon I, Moro E, Grimaldi A, Moretto P, Baj A, Giron MC, Karousou 595 E, Viola M, Crema F, Frigo G, Passi A, Giaroni C, Vigetti D. 2017b. Changes in hyaluronan 596 deposition in the rat myenteric plexus after experimentally-induced colitis. Scientific Reports 597 7(1):17644.

598 Giaroni C, Marchet S, Carpanese E, Prandoni V, Oldrini R, Bartolini B, Moro E, Vigetti D, 599 Crema F, Lecchini S, Frigo G. 2013. Role of neuronal and inducible nitric oxide synthases in the 600 guinea pig ileum myenteric plexus during in vitro ischemia and reperfusion. 601 Neurogastroenterology \& Motility 25(2):e114-126.

602 Giaroni C, Zanetti E, Giuliani D, Oldrini R, Marchet S, Moro E, Borroni P, Trinchera M,

603 Crema F, Lecchini S, Frigo G. 2011. Protein kinase C modulates NMDA receptors in the 604 myenteric plexus of the guinea pig ileum during in vitro ischemia and reperfusion. 605 Neurogastroenterology \& Motility 23(2):e91-103.

606 Giaroni C. Purinergic signalling and development of the autonomic nervoussystem. 2015. 607 Autonomic Neuroscience 191:67-77.

608 Housset M, Samuel A, Ettaiche M, Bemelmans A, Béby F, Billon N, Lamonerie T. 2013. Loss 609 of Otx2 in the adult retina disrupts retinal pigment epithelium function, causing photoreceptor 610 degeneration. Journal of Neuroscience 33(24):9890-904.

611 Joshi RL, Torero Ibad R, Rheey J, Castagner F, Prochiantz A, Moya KL. 2011. Cell non612 autonomous functions of homeoproteins in neuroprotection in the brain. FEBS Letters 613 585(11):1573-1578.

614 Kastury K, Druck T, Huebner K, Barletta C, Acampora D, Simeone A, Faiella A, Boncinelli 615 E. 1994. Chromosome locations of human EMX and OTX genes. Genomics. 22(1):41-45.

616 Kobayashi H, Kusafuka J, Lane GJ, Yamataka A, Satoh K, Hayakawa T, Kase Y, Hatano 617 M. 2007. The mechanism of intestinal motility in homozygous mutant Ncx/Hox11L.1-deficient 618 mice--a model for intestinal neuronal dysplasia. Journal of Pediatric Surgery 42(12):2062-2066.

619 Lakhan SE, Kirchgessner A. 2010. Neuroinflammation in inflammatory bowel disease. Journal 620 of Neuroinflammation 7:37.

621 Larsen KB, Lutterodt MC, Møllgård K, Møller M. 2010. Expression of the homeobox genes 622 OTX2 and OTX1 in the early developing human brain. Journal of Histochemistry and 623 Cytochemistry 58(7):669-678.

624 Leite RF, Annes K, Ispada J, de Lima CB, Dos Santos ÉC, Fontes PK, Nogueira MFG, 625 Milazzotto MP. 2017. Oxidative Stress Alters the Profile of Transcription Factors Related to Early 626 Development on In Vitro Produced Embryos. Oxidative Medicine and Cellular Longevity 6271502489.

628 Liu S, Xia Y, Hu Hz, Ren J, Gao C, Wood JD. 2000. Histamine H3 receptor-mediated 629 suppression of inhibitory synaptic transmission in the submucous plexus of guinea-pig small 630 intestine. European Journal of Pharmacology 397(1):49-54. 
631 Lomax AE, Fernández E, Sharkey KA. 2005 Plasticity of the enteric nervous system during

632

633

634

635

636

637

638

639

640

641

642

643

644

645

646

647

648

649

650

651

652

653

654

655

656

657

658

659

660

661

662

663

664

665

666

667

668

669

670

671

672

intestinal inflammation. Neurogastroenterology \& Motility 17(1):4-15.

Mathis T, Housset M, Eandi C, Beguier F, Touhami S, Reichman S, Augustin S, Gondouin P, Sahel JA, Kodjikian L, Goureau O, Guillonneau X, Sennlaub F. 2017. Activated monocytes resist elimination by retinal pigment epithelium and downregulate their OTX2 expression via TNF- $\alpha$. Aging Cell 16(1):173-182.

Moreels TG, De Man JG, De Winter BY, Timmermans JP, Herman AG, Pelckmans PA. 2001. Effect of 2,4,6-trinitrobenzenesulphonic acid (TNBS)-induced ileitis on the motor function of non-inflamed rat gastric fundus. Neurogastroenterology \& Motility 13(4):339-352.

Mourad FH, Hamdi T, Barada KA, Saadé NE. 2016. Experimental colitis in rats induces de novo synthesis of cytokines at distant intestinal sites: role of capsaicin-sensitive primary afferent fibers. European Cytokine Network 27(2):41-53.

Neurath MF. Current and emerging therapeutic targets for IBD. 2017. Nature Reviews in Gastroenterology and Hepatology 14(5):269-278

Ng SC, Shi HY, Hamidi N, Underwood FE, Tang W, Benchimol EI, Panaccione R,Ghosh S, Wu JCY, Chan FKL, Sung JJY, Kaplan GG. 2018. Worldwide incidence and prevalence of inflammatory bowel disease in the 21 st century: a systematic review of population-based studies. Lancet 390(10114):2769-2778.

Ni J, Wu GD, Albenberg L, Tomov VT. 2017. Gut microbiota and IBD: causation or correlation? Nature Reviews in Gastroenterology and Hepatolog 14(10):573-584.

\section{Pagano E, Capasso R, Piscitelli F, Romano B, Parisi OA, Finizio S, Lauritano}

\section{A, Marzo VD, Izzo AA, Borrelli F. 2016. An Orally Active Cannabis Extract with High} Content in Cannabidiol attenuates Chemically-induced Intestinal Inflammation and Hypermotility in the Mouse. Frontiers in Pharmacology 7:341.

Pagano E, Romano B, Iannotti FA, Parisi OA, D'Armiento M, Pignatiello S, Coretti L, Lucafò M, Venneri T, Stocco G, Lembo F, Orlando P, Capasso R, Di Marzo V, Izzo AA, Borrelli F. 2019. The non-euphoric phytocannabinoid cannabidivarin counteracts intestinal inflammation in mice and cytokine expression in biopsies from UC pediatric patients. Pharmacological Research 149:104464.

Puelles E, Acampora D, Gogoi R, Tuorto F, Papalia A, Guillemot F, Ang SL,Simeone A. 2006. Otx2 controls identity and fate of glutamatergic progenitors of the thalamus by repressing GABAergic differentiation. Journal of Neuroscience 26(22):5955-5964.

Sherf O, Nashelsky Zolotov L, Liser K, Tilleman H, Jovanovic VM, Zega K, Jukic MM, Brodski C. 2015. Otx2 Requires Lmx1b to Control the Development of Mesodiencephalic Dopaminergic Neurons. PLoS One 10(10):e0139697.

Spatazza J, Di Lullo E, Joliot A, Dupont E, Moya KL, Prochiantz A. 2013. Homeoprotein signaling in development, health, and disease: a shaking of dogmas offers challenges and promises from bench to bed. Pharmacological Reviews 65(1):90-104.

Szebeni GJ, Nagy LI, Berkó A, Hoffmann A, Fehér LZ, Bagyánszki M, Kari B, Balog JA, Hackler L Jr, Kanizsai I, Pósa A, Varga C, Puskás LG. 2019. The Anti-Inflammatory Role of Mannich Curcuminoids; Special Focus on Colitis. Molecules 24(8).

Peer] reviewing PDF | (2019:08:40795:1:1:NEW 10 Dec 2019) 
673 Terrinoni A, Pagani IS, Zucchi I, Chiaravalli AM, Serra V, Rovera F, Sirchia S, Dionigi G,

674 Miozzo M, Frattini A, Ferrari A, Capella C, Pasquali F, Curto FL, Albertini A, Melino G,

675 Porta G. 2011. OTX1 expression in breast cancer is regulated by p53. Oncogene 30(27):3096-

676103.

677 Thrasivoulou C, Soubeyre V, Ridha H, Giuliani D, Giaroni C, Michael GJ, Saffrey MJ,

678 Cowen T. 2006. Reactive oxygen species, dietary restriction and neurotrophic factors in age679 related loss of myenteric neurons. Aging Cell 5(3):247-57.

680 Zhang T, Chen H, Qi L, Zhang J, Wu R, Zhang Y, Sun Y. 2018 Transcript Profiling 681 Identifies Early Response Genes against FMDV Infection in PK-15 Cells. Viruses 10: 7.

682 Yu K, Cai XY, Li Q, Yang ZB, Xiong W, Shen T, Wang WY, Li YF. 2014. OTX1 promotes

683 colorectal cancer progression through epithelial-mesenchymal transition. Biochemical Biophysical 684 Research Communication 444(1):1-5. 


\section{Figure 1}

Histological evaluation, OTX1 and OTX2 staining in small intestine and colonic cross sections after DNBS-induced colitis.

Hematoxylin-eosin (HE) staining highlights the well-preserved cellular and structural morphology of small intestine (A) and distal colon (K) cross-sections of control (vehicletreated, CTR) rats (bar: $50 \mu \mathrm{m}$ ). Detail of a myenteric ganglion in cross-sections obtained from small intestine (B) and colon (L) of CTR rats (bars: $10 \mu \mathrm{m}$ ). Both in the small intestine (C) and distal colon (M) of CTR animals, CD45 staining, as an index of inflammatory infiltrate, was slight (bars: $10 \mu \mathrm{m}$ ). After DNBS treatment no significant morphological changes were evident in the small intestine $(\mathbf{F})$, while important structural changes were observed in the distal colon (P), including increased thickness of the muscularis mucosae, submucosal layer and muscularis propria layers (bars: $50 \mu \mathrm{m}$ ). Detail of a myenteric plexus ganglion in small intestine $(\mathbf{G})$ and distal colon $(\mathbf{R})$ cross-sections obtained after DNBS treatment (bars: 10 $\mu \mathrm{m})$. In particular, HE staining highlights prominent distortions in a colonic myenteric plexus ganglion (R). Panel $\mathbf{Q}$ shows the presence of spaces among smooth muscle cells and leukocytes infiltrates (arrowheads) in a colonic cross-section after DNBS treatment (bar 10 $\mu \mathrm{m})$. Co-staining with the pan neuronal marker HuC/D, showed few myenteric neurons labelling for both OTX1 and OTX2 (arrows) in the small intestine (D, N) and colon (E, O) of CTR rats, inserts show details of myenteric ganglia (bars: $10 \mu \mathrm{m}$; inserts $5 \mu \mathrm{m}$ ). After DNBS treatment, both OTX1 and OTX2 immunoreactivity increased in myenteric neurons of both regions (small intestine: $\mathbf{I}, \mathbf{T}$; distal colon: J, U; bars: $10 \mu \mathrm{m}$, inserts $5 \mu \mathrm{m}$ ). Arrows indicate myenteric plexus ganglia. CM, circular muscle; LM, longitudinal muscle; MP, myenteric plexus; M, mucosa; SM, submucosa; Mus, Muscularis Propria. 

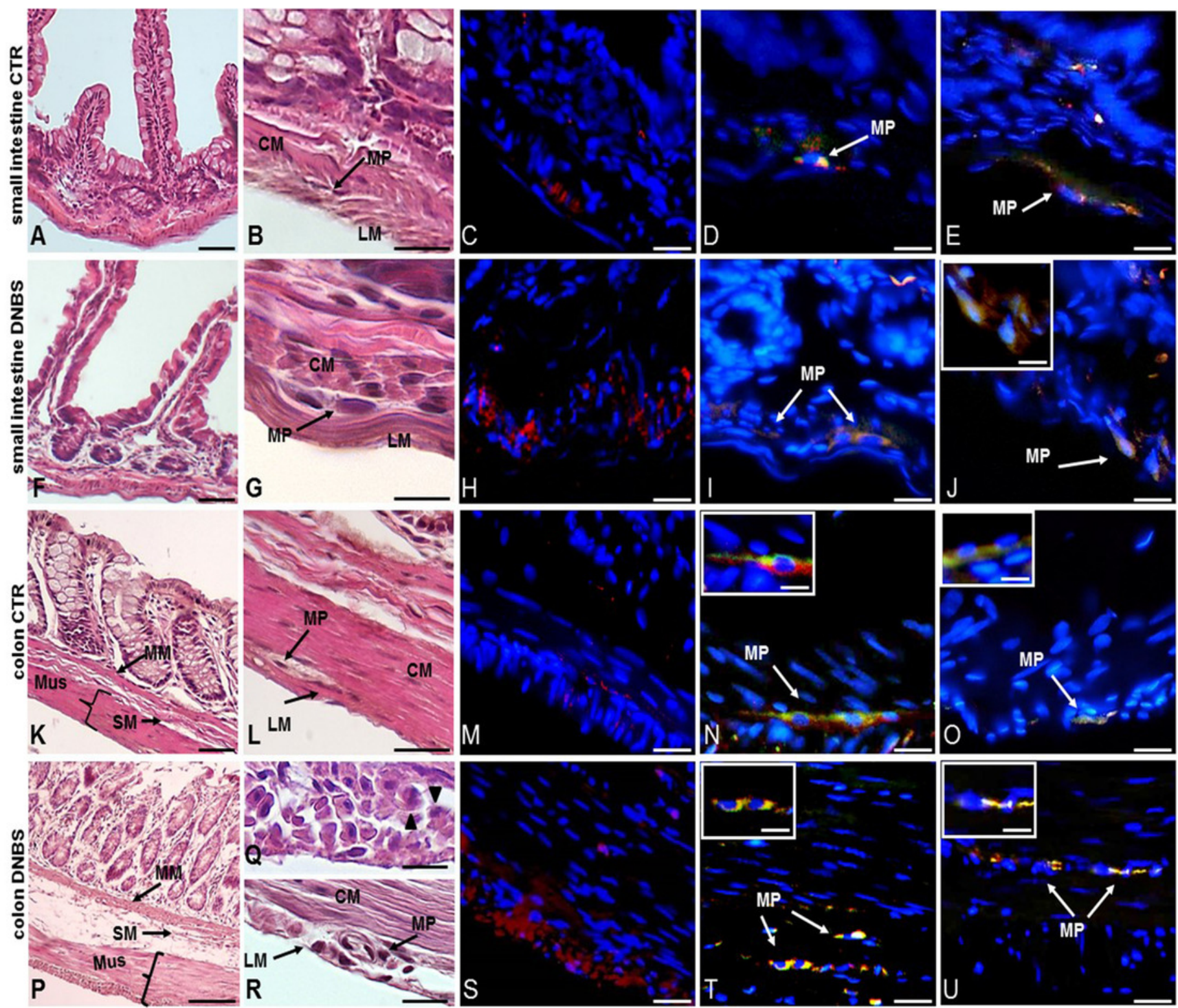


\section{Figure 2}

DNBS treatment-induced changes in myenteric neurons.

(A-D) HuC/D staining of myenteric neurons in CTR and DNBS-treated whole-mount LMMP preparations. (E) Myenteric neuron number normalized per ganglion area in small intestine and distal colon LMMP whole-mount preparations obtained from DNBS-treated (solid bar) and control animals (vehicle-treated CTR, empty bar). (F) Mean myenteric neuron area calculated in small intestine and distal colon whole-mount preparations obtained from DNBS-treated (solid bar) and control animals (vehicle-treated CTR, empty bar). Values are expressed as mean \pm S.E.M. $N=5$ rats per group. $* P<0.05$ and ${ }^{* * * P}<0.001$ vs values obtained in CTR animals by One Way ANOVA with Tukey's post hoc test. ${ }^{\circ} \mathrm{P}<0.05$ vs CTR small intestine. $\S \mathrm{P}<0.05$ vs DNBS-treated small intestine. 

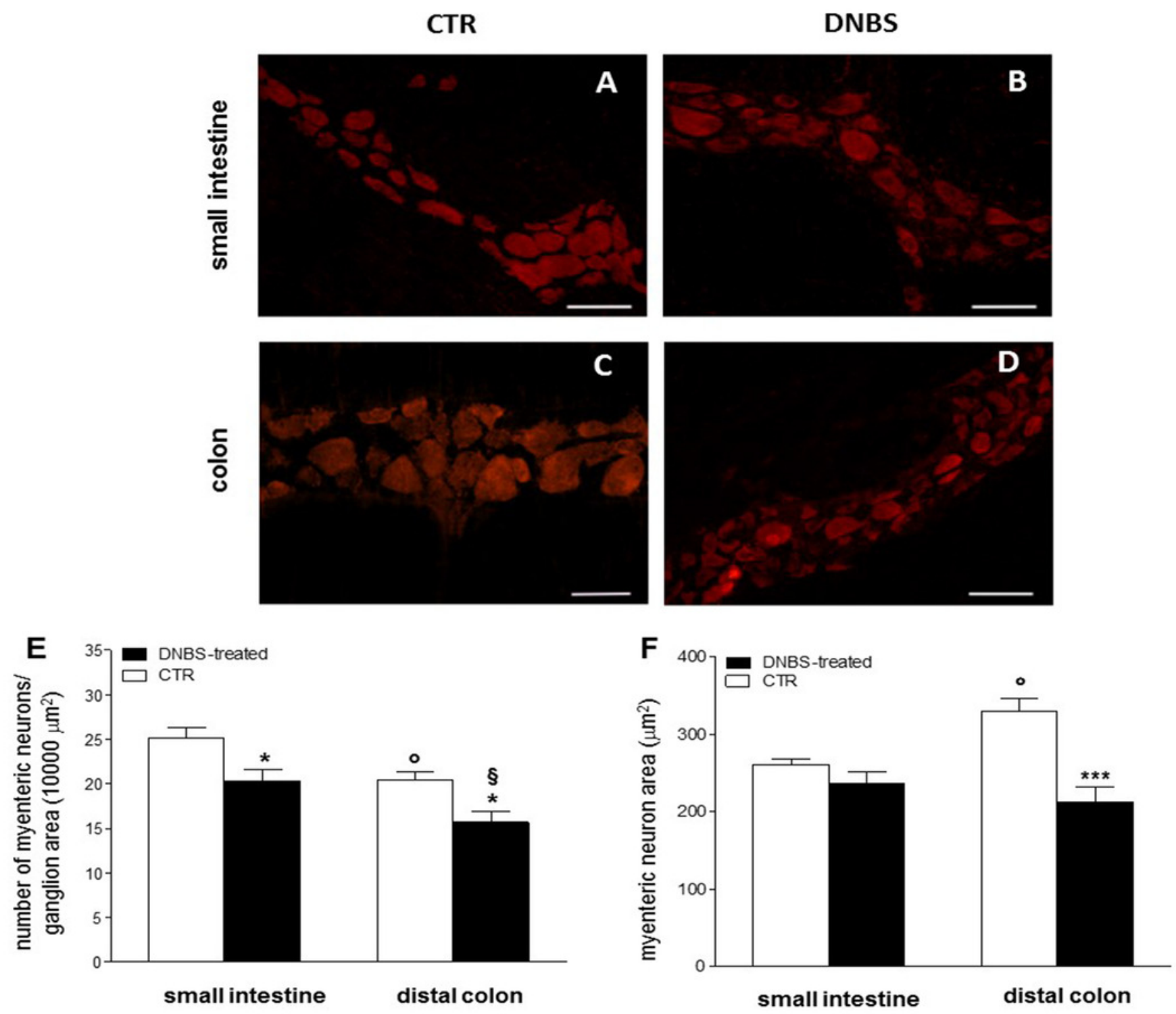


\section{Figure 3}

Expression of Inflammatory markers in the ileum and colon after DNBS-induced colitis.

(A-E) qRT-PCR quantification of TNF $\alpha$, pro-IL1 $\beta$, IL6, HIF1 $\alpha$, VEGF $\alpha$ mRNA levels obtained in the small intestine and distal colon of control animals (vehicle-treated, CTR) and after DNBS treatment (solid bars). The relative gene expression was determined by comparing $2^{-\Delta \Delta C t}$ values in CTR and DNBS-treated samples normalized to $\beta$-actin. Values are mean $\pm S$.E.M. of at least 7-9 experiments. $* P<0.05, * * P<0.01$ and $* * *<<0.0001$ vs values obtained in control animals; ${ }^{\S} \mathrm{P}<0.05$ and ${ }^{\$ S \mathrm{P}} \mathrm{P}<0.001$ vs DNBS-treated small intestine. Significance was evaluated by one-way ANOVA with Tukey's post hoc test. (F) MPO activity measured in mucosadeprived small intestine and distal colon segments obtained from DNBS-treated (solid bar) and control animals (vehicle-treated, CTR). Values are expressed as mean \pm S.E.M. of 6 experiments. ${ }^{* * * P}<0.001$ vs values obtained in control animals, by one-way ANOVA with Tukey's post hoc test. 

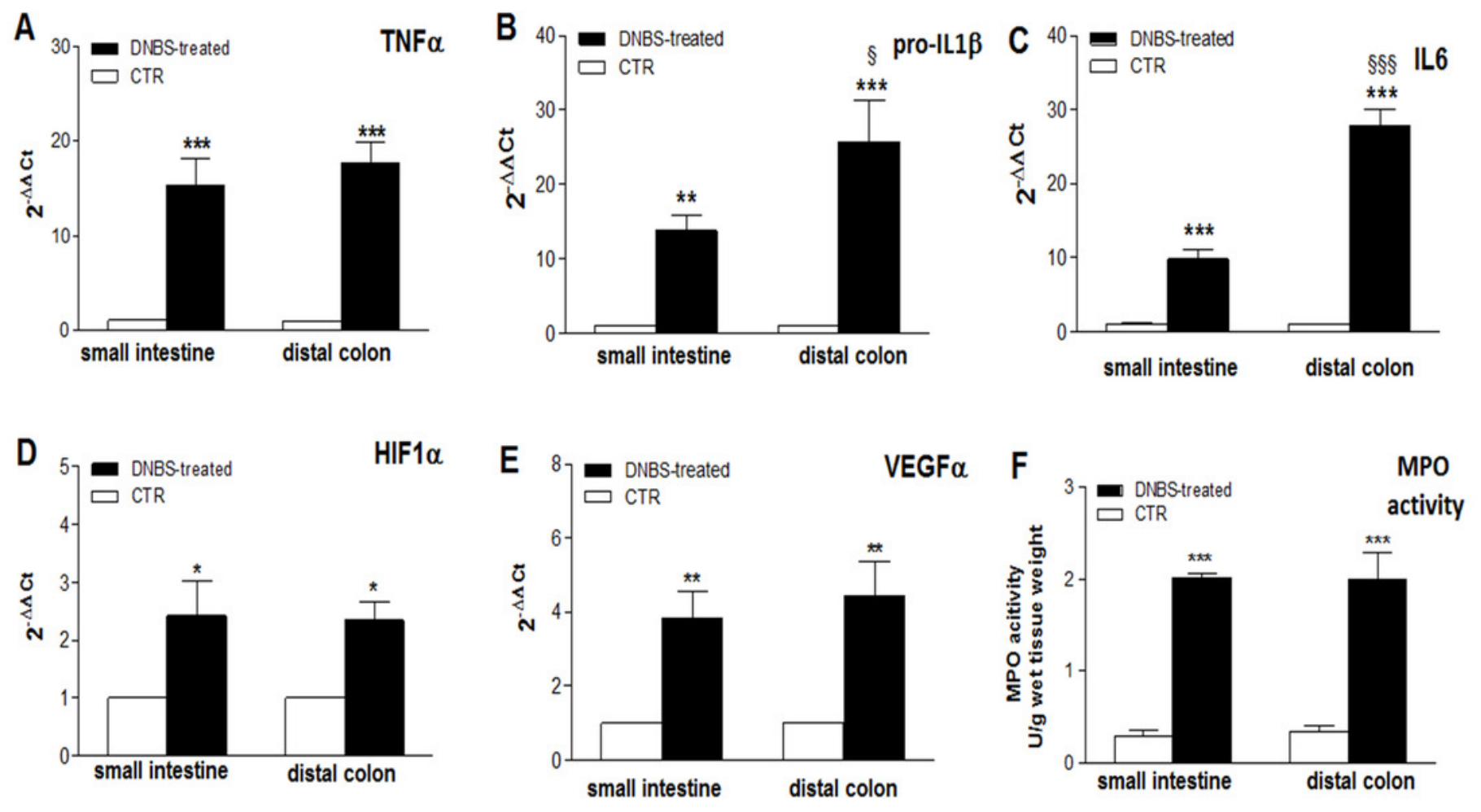


\section{Figure 4}

Percentage of OTX1 and OTX2 neurons in the rat small intestine and distal colon after DNBS-treatment.

(A) Percentage of OTX1 immunoreactive myenteric neurons with respect to the total of HuC/D immunoreactive neurons per ganglion in small intestine and distal colon LMMPs wholemount preparations obtained from DNBS-treated (solid bar) and control animals (vehicletreated CTR, empty bar). (B) Percentage of OTX2 immunoreactive myenteric neurons with respect to the total of HuC/D immunoreactive neurons per ganglion in small intestine and distal colon whole-mount preparations obtained from DNBS-treated (solid bar) and control animals (vehicle-treated CTR, empty bar). Values are expressed as mean $\pm S . E . M, N=5$ rat per group. ${ }^{* * *} \mathrm{P}<0.001$ vs values obtained in CTR animals, ${ }^{\$ \varsigma \varsigma} \mathrm{P}<0.001$ vs DNBS-treated small intestine by one-way ANOVA with Tukey's post hoc test.
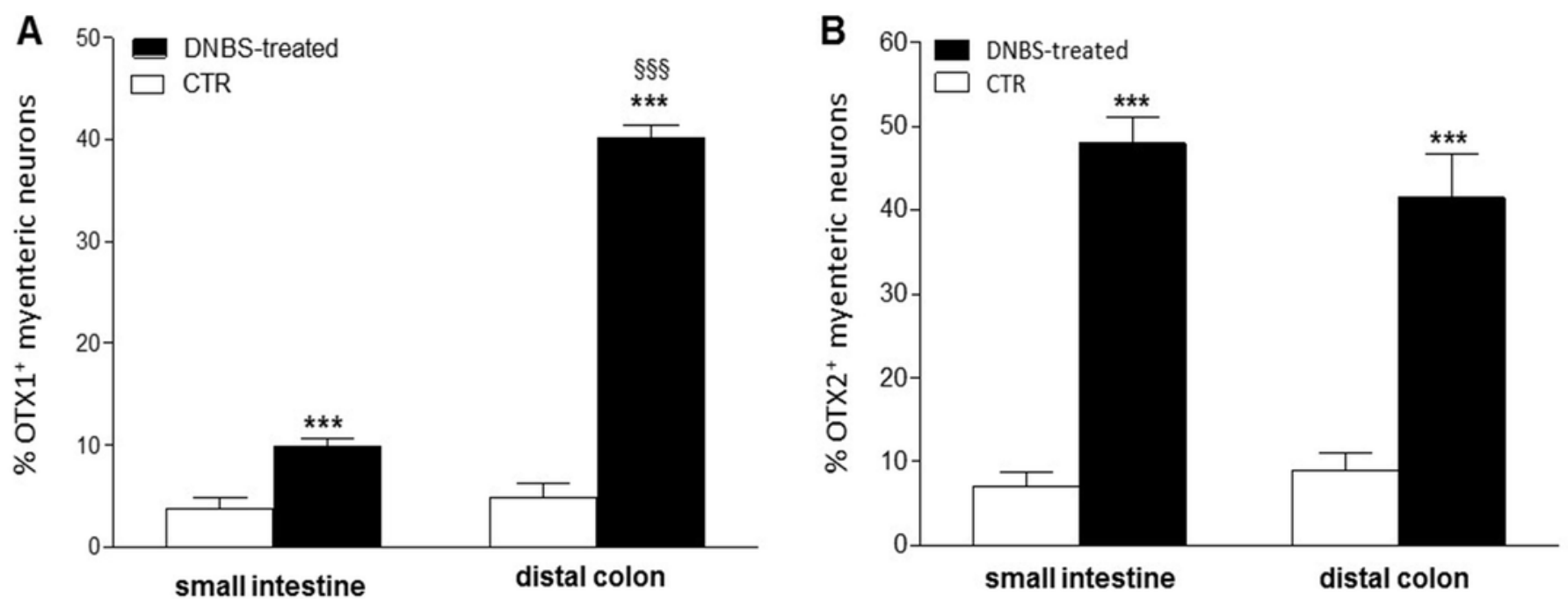


\section{Figure 5}

OTX1 localization in the rat small intestine and distal colon myenteric plexus of CTR and DNBS-treated rats.

Co-localization of OTX1 with the neuronal marker HuC/D in LMMPs whole-mount preparations of the small intestine and distal colon obtained from vehicle-treated CTR animals (A-C; G-L, respectively) and DNBS-treated animals (D-F; J-L, respectively). In CTR preparations few neurons displayed OTX1 immunoreactivity (arrow) (A-C; G-I). In both regions, the number of OTXI-IR neurons was higher in DNBS-treated preparations (D-F; J-L). Photomicrographs of small intestine (M-O) and colonic (P-R) LMMP whole-mount preparations showing OTX1 immunoreactivity in enteric glial cells (asterisk) as evidenced by co-staining with the glial cell marker, S100ß. Bar $50 \mu \mathrm{m}$. 


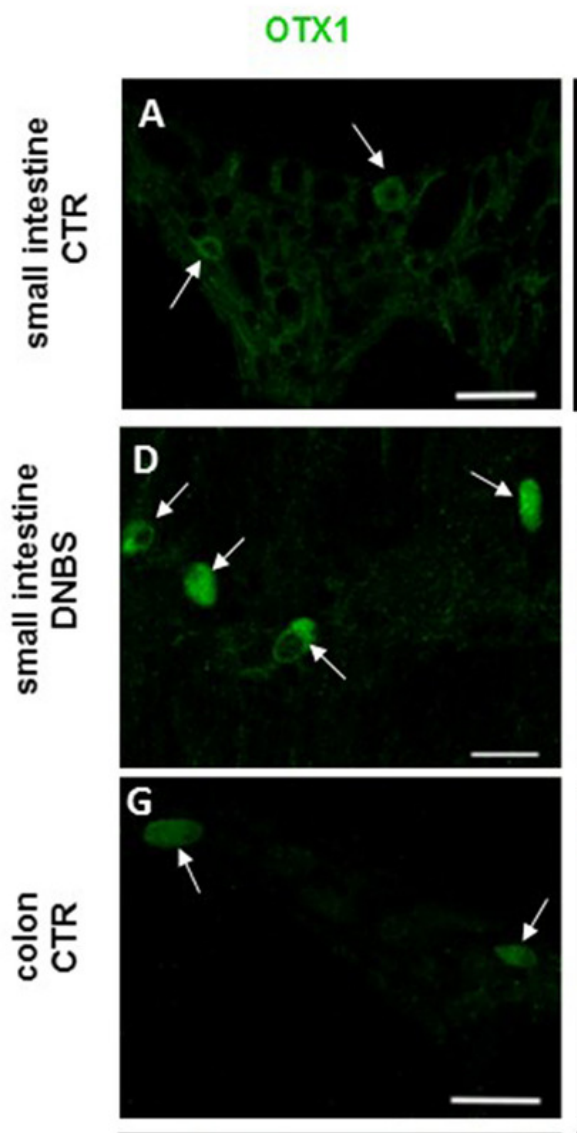

$\mathrm{HuClD}$
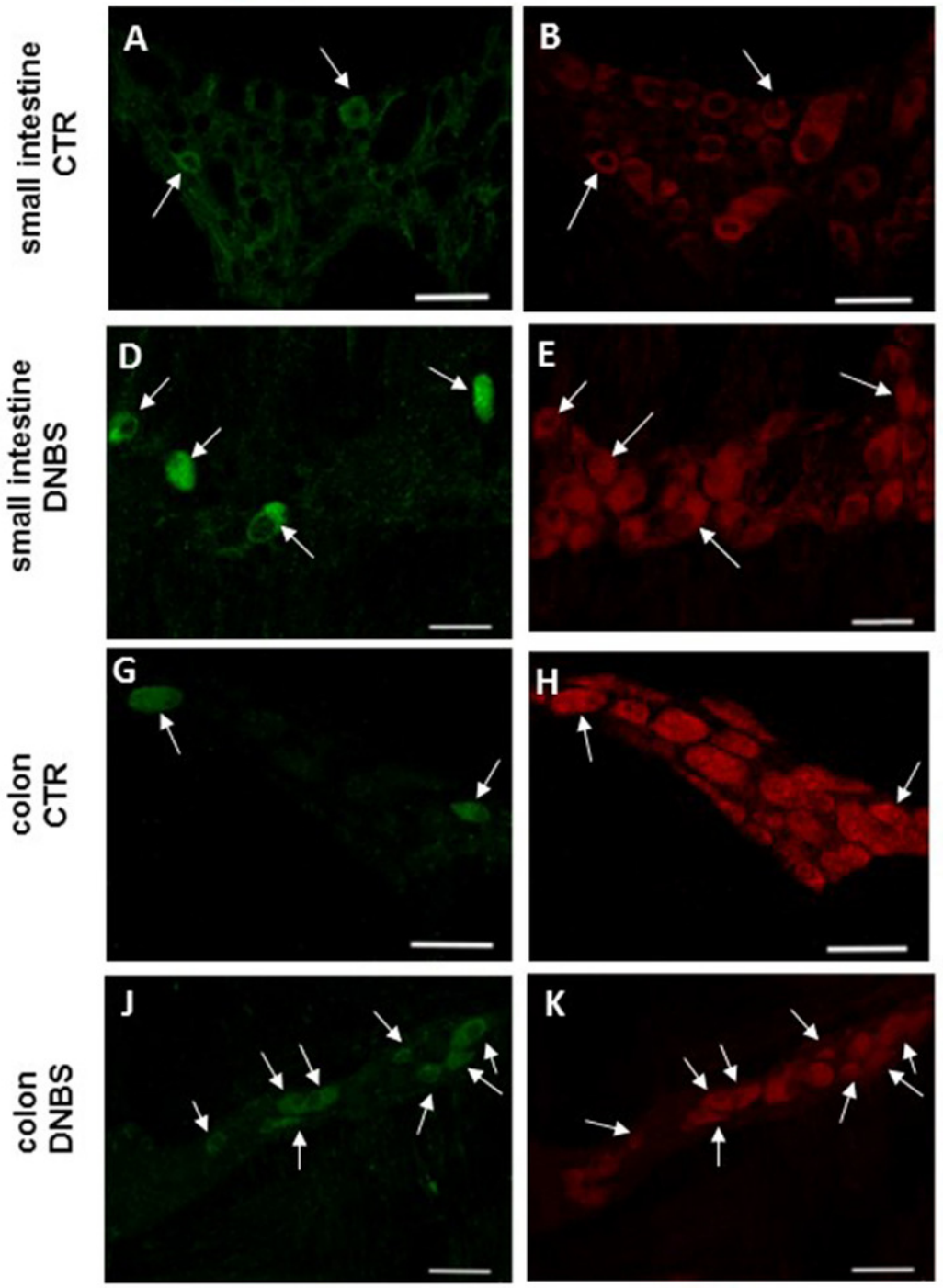

$S 100 \beta$
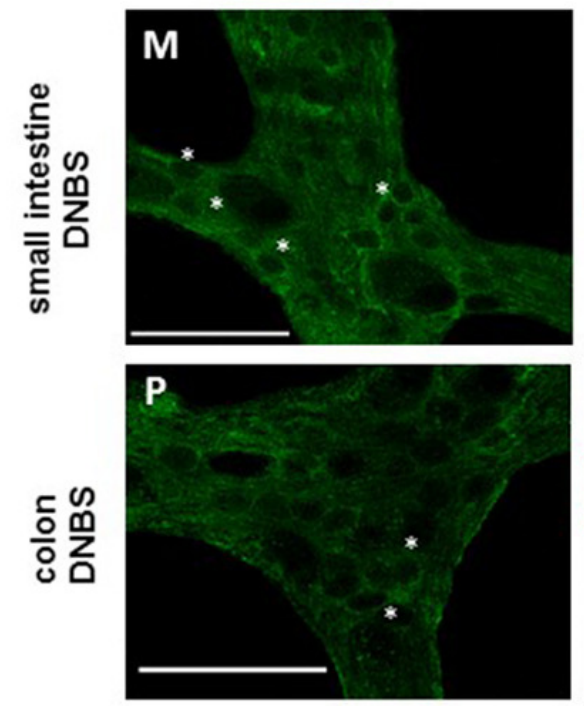

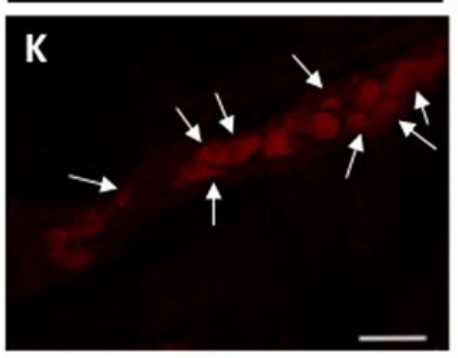

OTX1

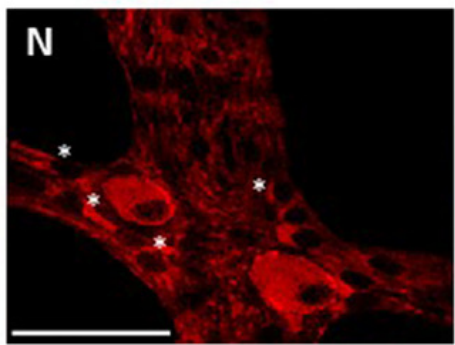

Q

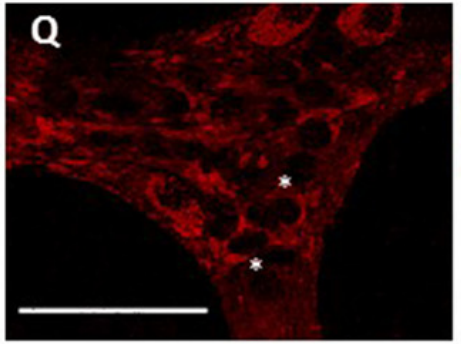

OTX1HuC/D
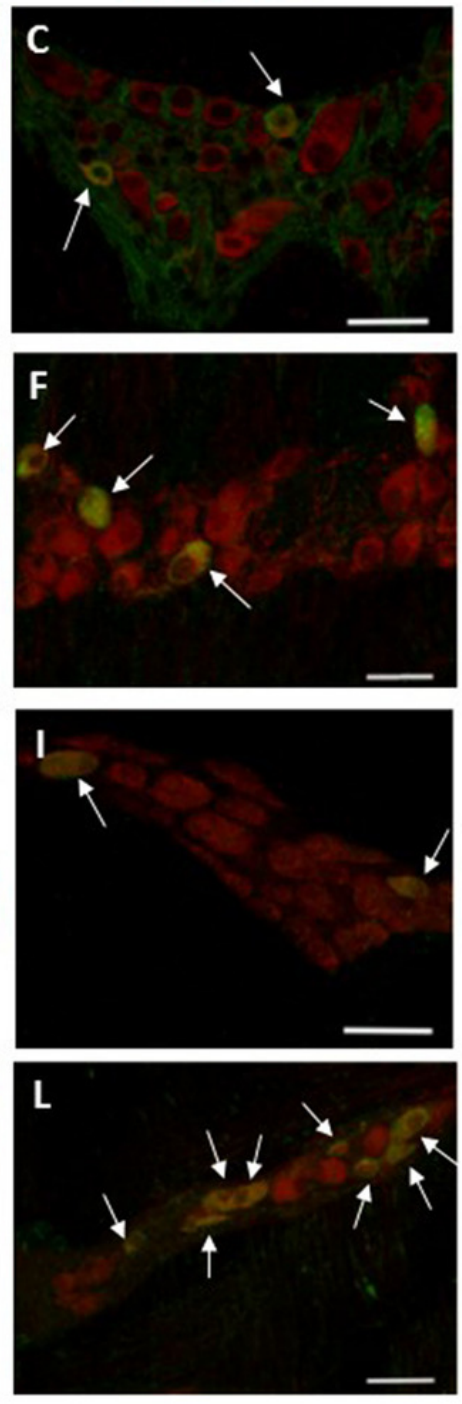

S100B OTX1

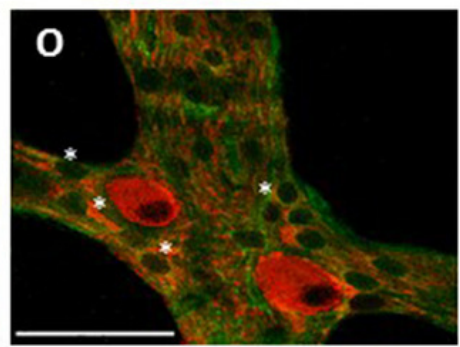

$\mathbf{R}$ 


\section{Figure 6}

OTX1 and iNOS co-localization in the rat small intestine and distal colon myenteric plexus of CTR and DNBS-treated rats.

Photomicrograph showing few myenteric neurons (arrow) co-staining OTX1 and INOS in CTR LMMPs whole-mount preparations of the small intestine (A-C) and distal colon (G-I). After DNBS treatment, the number of iNOS immunoreactive myenteric neurons (arrow) increased and co-expressed OTX1 both in small intestine (D-F) and distal colon (J-L). Both in CTR and DNBS-treated group, co-staining between OTX1 and nNOS was not detected in myenteric ganglia of both small intestine and distal colon. A colonic whole-mount LMMP preparation obtained from DNBS-treated animals is reported as an example (M-O, bar: $50 \mu \mathrm{m})$ bar: 50 $\mu \mathrm{m}$ 


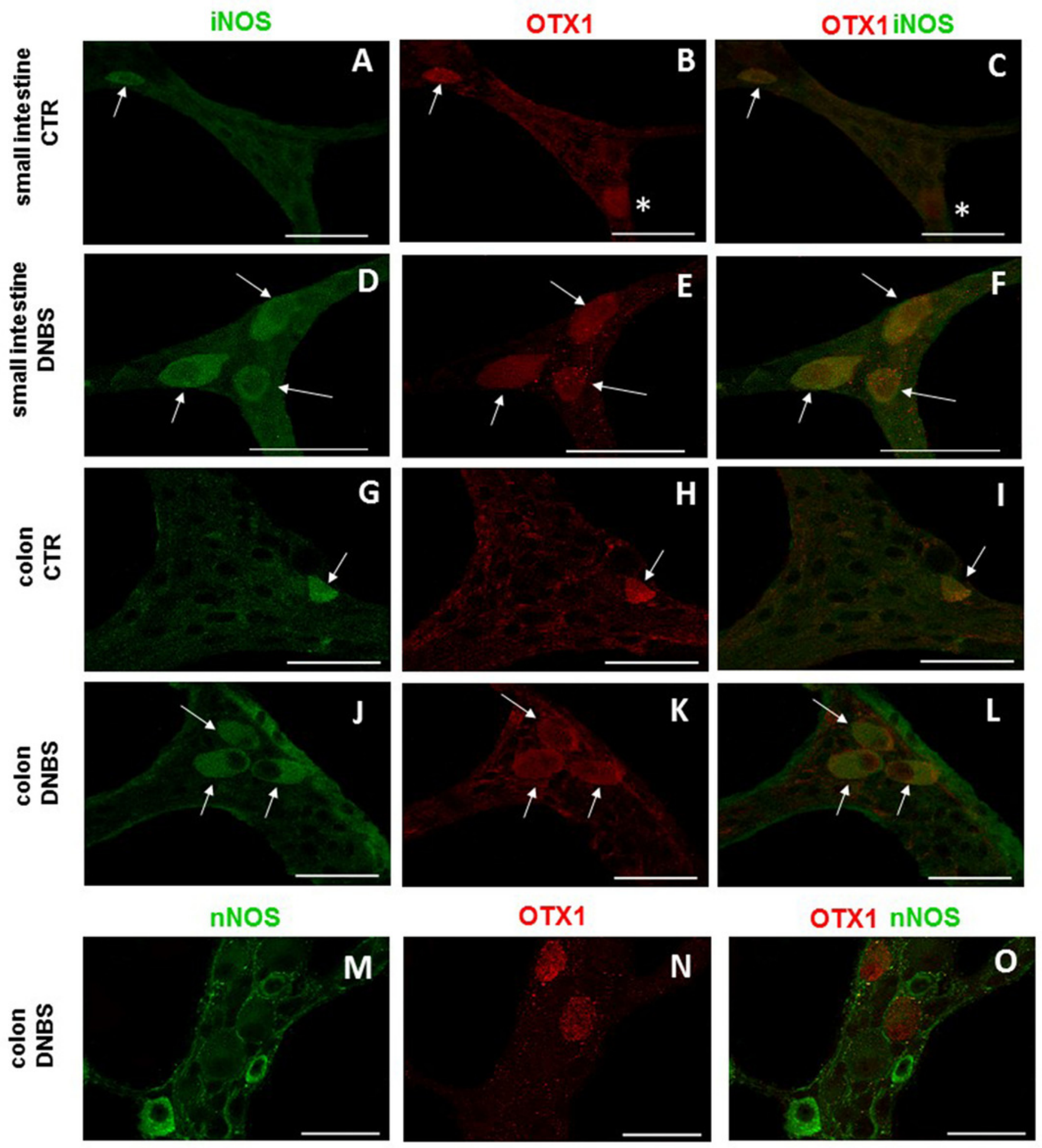




\section{Figure 7}

OTX2 localization in the rat small intestine and distal colon myenteric plexus of CTR and DNBS-treated rats

Co-localization of OTX2 with the neuronal marker HuC/D in LMMPs whole-mount preparations of the small intestine and distal colon obtained from vehicle-treated CTR (A-C; G-I, respectively) and DNBS-treated animals (D-F; J-L, respectively). In CTR preparations, few neurons displayed OTX2 immunoreactivity (arrow). In both regions, after DNBS treatment, the number of OTX2 immunoreactive neurons significantly increased. Bar $50 \mu \mathrm{m}$. 

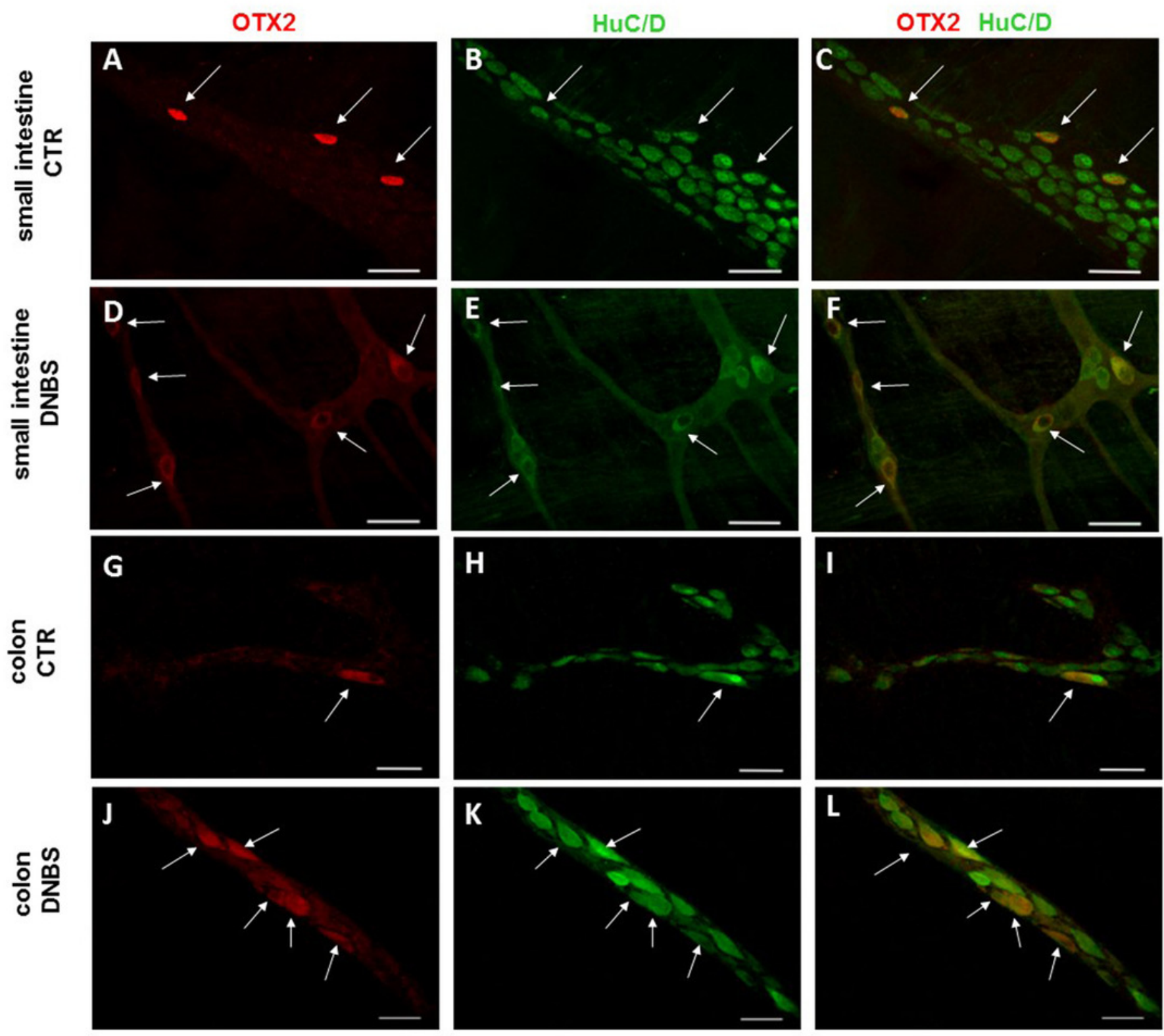


\section{Figure 8}

OTX2 and nNOS co-localization in the rat small intestine and distal colon myenteric plexus of CTR and DNBS-treated rats.

In myenteric neurons of both small intestine and distal colon from CTR and DNBS-treated rats, OTX2 co-localized with nNOS (arrows). Some OTX2 immunoreactive neurons, however, did not stain for nNOS (asterisk) (A-L, bars: $50 \mu \mathrm{m}$ ). Co-staining between OTX2 and iNOS was not detected, in myenteric ganglia of both small intestine and distal colon of both experimental groups. A colonic whole-mount LMMP preparation obtained from DNBS-treated animals is reported as an example (M-O, bar: $50 \mu \mathrm{m})$. 


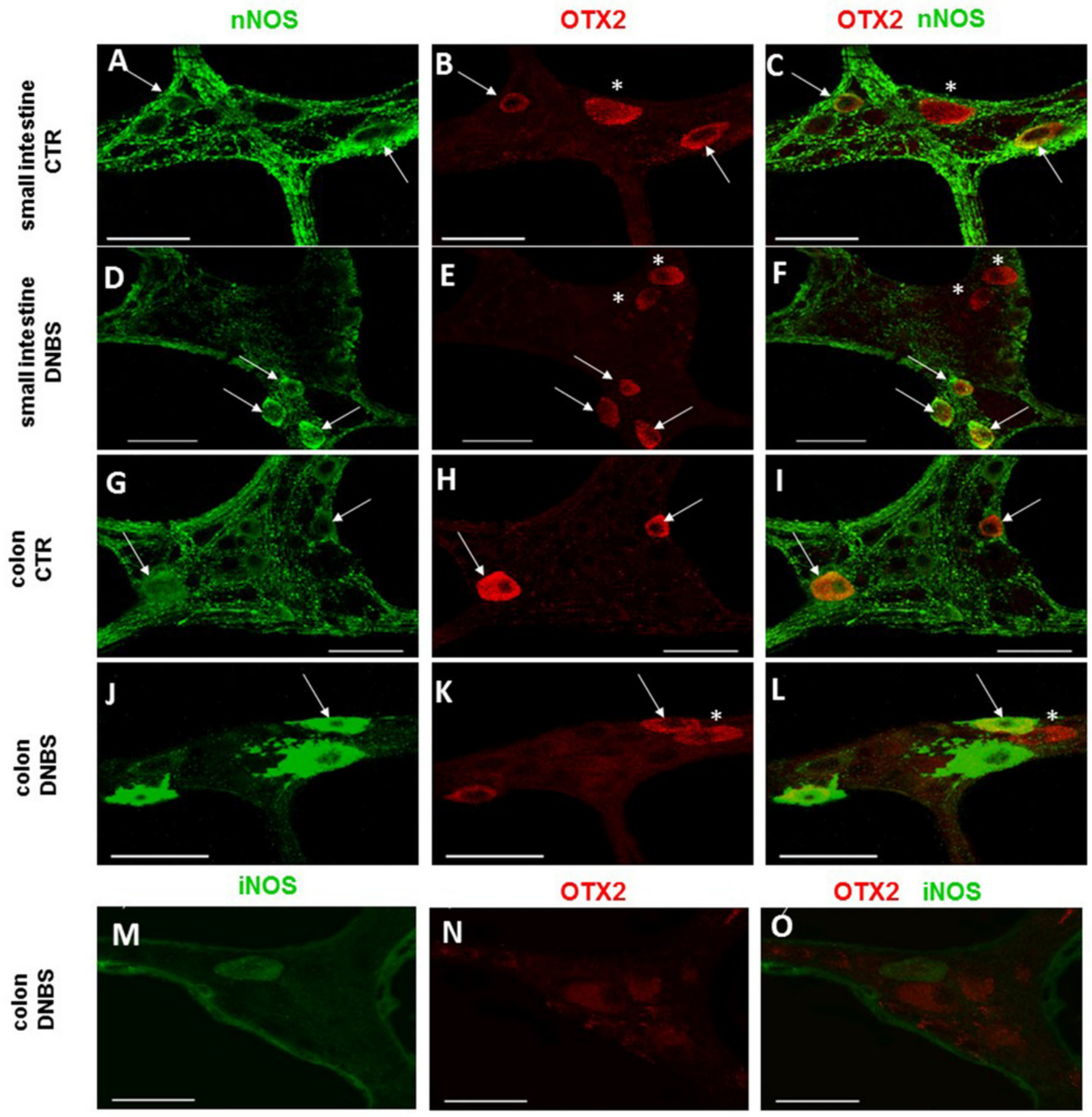




\section{Figure 9}

qRT-PCR and western blot analysis of OTX1 and OTX2 mRNA and protein levels in the rat small intestine and colon after DNBS-induced colitis.

RT-PCR quantification of OTX1 (A) and OTX2 (C) transcripts in preparations of the small intestine and distal colon obtained from control (CTR, empty bars) and DNBS-treated animals (solid bars). The relative gene expression was determined by comparing $2^{-\Delta \Delta C t}$ values in CTR and DNBS-treated samples normalized to $\beta$-actin. Values are mean \pm S.E.M. of 7-13

experiments. ${ }^{* P}<0.05,{ }^{* * * P}<0.001$ vs CTR animals; ${ }^{\$ \S} \mathrm{P}<0.001$ vs DNBS-treated small intestine by one- way ANOVA with Tukey's post hoc test. (B) OTX1 and (D) OTX2 protein expression analyzed in LMMPs preparations of the small intestine and colon obtained from CTR (empty bars) and DNBS-treated animals (solid bars). Blots representative of immunoreactive bands for either OTX1 or and $\beta$-actin in the different experimental conditions are reported on top of each panel. Numbers at the margins of the blots indicate relative molecular weights of the respective protein in kDa. Samples $(200 \mu \mathrm{g})$ were electrophoresed in SDS-8\% polyacrylamide gels. Values are expressed as mean \pm S.E.M. of 5-6 experiments of the percentage variation of the normalized optical density (O.D.) obtained from DNBS-treated preparations with respect to values obtained in control samples. $* \mathrm{P}<0.05$ ad $* * \mathrm{P}<0.01$ vs CTR by one-way ANOVA with Tukey's post hoc test 

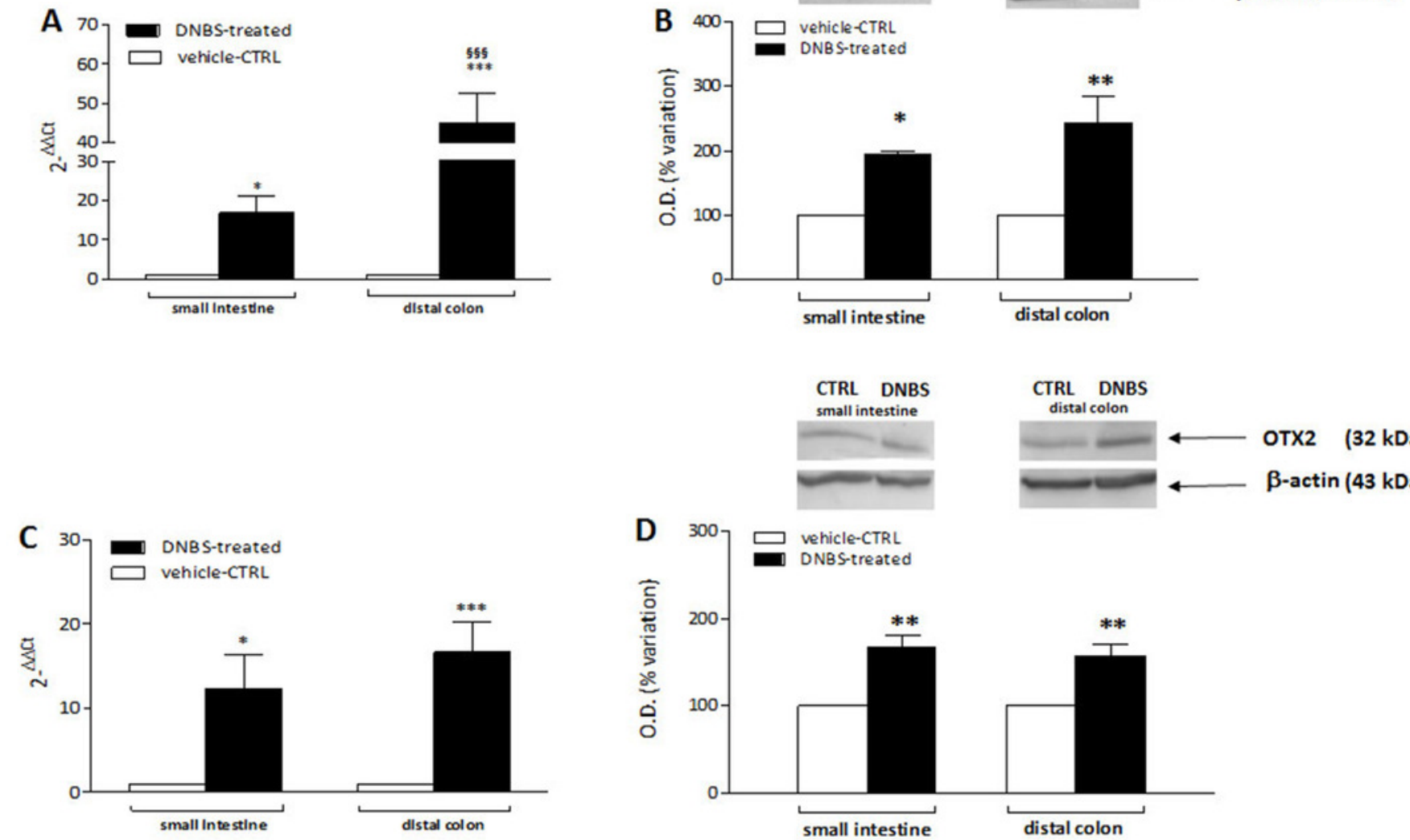


\section{Table $\mathbf{1}$ (on next page)}

Primary and secondary antisera and their respective dilutions used for Western Blot (WB) assay and immunohistochemistry (HC). 


\begin{tabular}{|c|c|c|c|c|}
\hline Antiserum & $\begin{array}{l}\text { Dilution } \\
\text { (WB) }\end{array}$ & $\begin{array}{l}\text { Dilution } \\
(\mathrm{HC})\end{array}$ & Source & Host species \\
\hline \multicolumn{5}{|l|}{ Primary antisera } \\
\hline OTX1 & & $1: 100$ & Invitrogen (PA5-67901) & Rabbit \\
\hline OTX1 & $1: 200$ & $1: 100$ & Santa Cruz (sc133872) & Rabbit \\
\hline OTX2 & $1: 100$ & + & R\&D systems (AF1979) & Goat \\
\hline OTX2 Alexa Fluor 594 conjugated & & $1: 100$ & $\begin{array}{l}\text { Bioss Antibodies (bs-11597R- } \\
\text { A594) }\end{array}$ & Rabbit \\
\hline HUC/D biotin & & 1: 100 & Invitrogen (16A11) & Mouse \\
\hline$\beta$-actin & $1: 1000$ & 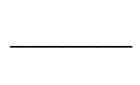 & $\begin{array}{l}\text { Cell Signalling Technology } \\
\text { (8H10D10) }\end{array}$ & Mouse \\
\hline nNOS & - & $1: 200$ & Millipore (AB1529) & Sheep \\
\hline nNOS & 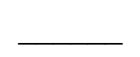 & $1: 50$ & Santa Cruz (sc648; R-20) & Rabbit \\
\hline iNOS & 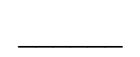 & $1: 50$ & Santa Cruz (sc8310; H-174) & Rabbit \\
\hline S100 & 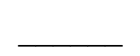 & $1: 100$ & Merck Millipore ( MAB079-1) & Mouse \\
\hline CD45 & 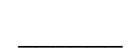 & $1: 100$ & Merck Millipore ( MAB079-1) & Mouse \\
\hline
\end{tabular}

Secondary antisera \& streptavidin complexes

\begin{tabular}{|c|c|c|c|c|}
\hline Anti-rabbit Alexa Fluor 488 & & $1: 200$ & Molecular Probes (A21206) & Donkey \\
\hline Anti-mouse Alexa Fluor 488 & & $1: 200$ & Molecular Probes (A21202) & Donkey \\
\hline Cy3-conjugated streptavidin & $\underline{-}$ & $1: 500$ & Amersham (PA43001) & \\
\hline FITC-conjugated straptavidin & 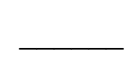 & $1: 200$ & Molecular Probes (SA1001) & \\
\hline $\begin{array}{l}\text { Anti-rabbit IgG HRP peroxidase } \\
\text { conjugated }\end{array}$ & $1: 5000$ & & Santa Cruz (sc2004) & Goat \\
\hline $\begin{array}{l}\text { Anti-goat IgG HRP peroxidase } \\
\text { conjugated }\end{array}$ & 1:10000 & & Santa Cruz (sc2020) & Donkey \\
\hline Anti-mouse IgG, HRP- linked & $1: 1000$ & & Cell Signalling Technology (\#7076) & Horse \\
\hline
\end{tabular}


Table 2 (on next page)

Sequence of primers used in the study for the analysis of qRT-PCR 


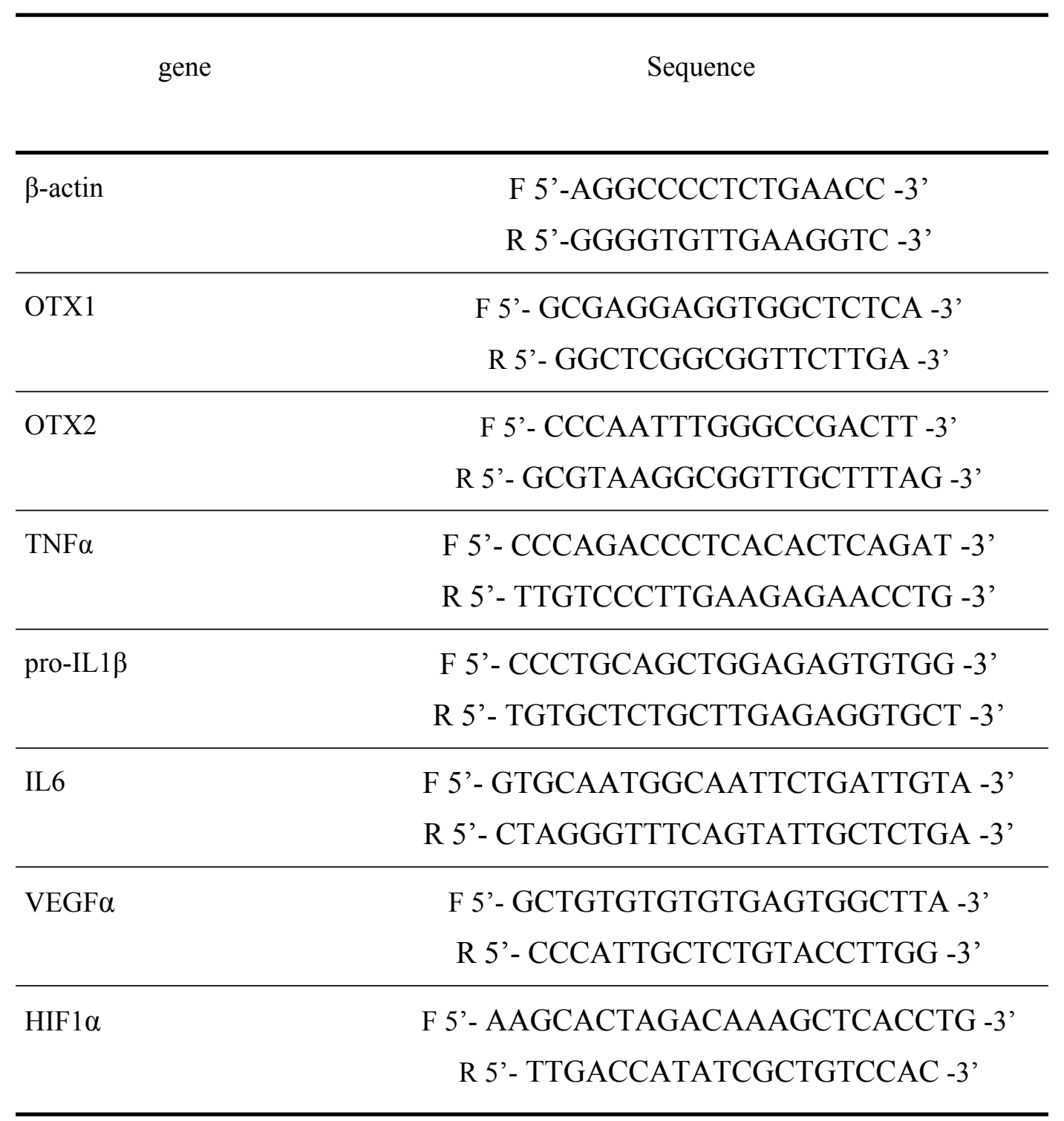

